\title{
Analysis of single crystalline microwires under torsion using a dislocation-based continuum formulation
}

\author{
Kolja Zoller ${ }^{\mathrm{a}}$, Katrin Schulz ${ }^{\mathrm{a}}$ \\ ${ }^{a}$ Karlsruhe Institute of Technology (KIT), Institute for Applied Materials - Computational Materials Science (IAM-CMS), \\ Kaiserstr. 12, 76131 Karlsruhe, Germany
}

\begin{abstract}
In the course of structural component miniaturization, the interest in the material behavior at small scales and underlying physical mechanisms has significantly grown in the last years. In this context, the consideration of dislocations plays an essential role for the analysis of the resulting plasticity and dislocation based simulations can give helpful insights into the material evolution. In this paper, a three-dimensional crystal plasticity continuum formulation is extended by a homogenized, mechanism based dislocation source model and applied to $\langle 100\rangle$ oriented single crystalline microwires under torsion. Fundamental operating principles of the individual slip systems, their interaction concerning the type of dislocations, relaxation mechanisms as well as the significance for the local misorientation are studied. Considering the torsion of a $24 \mu \mathrm{m}$ thick aluminum wire, the spatial distribution of strains and dislocation densities as well as the resultant local misorientation are shown compared to discrete dislocation dynamics simulations and experiments. The results show that characteristic spatial distributions of dislocation density over the cross section can be found. Due to internal dislocation pile-ups, a size effect for small microwires under torsion can be observed.
\end{abstract}

\section{Introduction}

Due to the miniaturization of structural components, the application for wires under torsion expands to small scales. Screws smaller than a millimeter are used for new smartphones, shafts of tiny motors reach sizes of a few hundred micrometers [40] and drills with a diameter of only few micrometers are used in medical technology. At micrometer scales, internal length scales have to be taken into account to capture the size effect observed for torsional loading [7, 41]. Furthermore, microwires under torsion are challenging due to complex physical mechanisms including internal pile-ups of dislocations in the presence of a linear external stress field and the interaction of different slip systems that are subjected to different resolved shear stress fields.

The torsion of microwires has been studied using different methods to analyze a number of different aspects. This includes molecular dynamics simulations examining the crystallographic orientation dependency of the deformation mechanisms and homogeneity of the plastic deformation [37, 38], discrete dislocation dynamics (DDD) simulations that were used to examine the dislocation microstructure evolution and the role of cross slip [32] as well as the crystallographic orientation and specimen size dependency of the plastic deformation [26]. Continuum dislocation analyses were used to study the dislocation density distribution considering energetic aspects for simplified artificial slip systems including dislocation nucleation and work hardening [13], as well as hardening and softening during the wire torsion and size effects [14]. Gradient plasticity formulations were used to analyze the relation between surface energy and boundary conditions [10], the plastic spin contribution [2], as well as the influence of texture on the size effect for oligocrystalls [3]. Basic theoretical considerations have been carried out to investigate the size effect at the onset of yielding and the distribution of stresses and geometric necessary dislocations (GNDs) [18], as well as the role of the accumulation and pile-ups of GNDs for the size effect [15]. To investigate the size effect of yielding and plastic flow due to a critical thickness effect and the role of GNDs, [17] performed experiments with polycrystalline microwires. For bamboo-structured microwires, [43] presented experimental results studying the deformation and misorientation of the structure.

Since plastic material behavior results from the evolution of the dislocation microstructure, the dislocation production plays an essential role. Considering several possible source mechanisms including Frank-Read sources, truncated sources, and dislocation nucleation from the surface, the governing source mechanisms can differ with varying sample size. Resulting effects on the initiation of yielding or due to the 'starvation' of mobile dislocations depending on the specimen size were observed e.g. in experiments [22, 8, 39]. Mathematical and numerical formulations have been proposed e.g. by [24, 6]. Source models for continuum formulations based on discrete dislocation mechanisms rely on the dislocation density production and additional microstructural information [25], as e.g. the dislocation velocities, type and length of involved dislocation segments or dimensions and amount of dislocation sources. Formulations using the nucleation time of a Frank-Read source have been introduced e.g. by [42, 21]. A model using an evolution parameter derived by discrete simulations is presented in [16]. However, it has been shown, that an inadequate parameterization of continuum models based on the nucleation time could lead to artificial softening [29]. Therefore, [25] takes into 
account a relaxation potential of the produced dislocations instead of using a rate formulation. An approach following the theory of critical thickness [5, 4] is realized by [29] for a twodimensional bending problem.

In this paper, the three-dimensional continuum dislocation dynamics (CDD) formulation according to [12, 31] is extended by a homogenized, mechanism based dislocation source model. Here, we focus on the averaged representation of dislocation source mechanisms corresponding to Frank-Read sources. Thus, the consideration of dislocation source activation allows for an increase of the total number of dislocations averaged in the continuum in contrast to the solely kinematic increase of dislocation density based on the increase of dislocation line length during the evolution of a number of existing dislocations in the system. We apply the formulation to a fcc microwire with $\langle 100\rangle$ orientation under torsion. The fundamental theoretical considerations and continuum simulations show the operating principles of the different slip systems including their interactions, relaxation mechanism and impact on the resulting dislocation microstructure and the misorientation of the continuum. The results are compared to DDD data and experimental investigations focusing on spatial distributions of dislocation densities and strains as well as the local misorientation over the wire cross section and along the wire axis.

\section{Methods}

\subsection{Dislocation density based continuum model}

Based on the CDD formulation in [31], we introduce an extension of this model incorporating a homogenized dislocation source formulation that enables e.g. the analysis of torsion problems from dislocation free initial structures. Although the basic set of CDD evolution equations is known from [31], the used formulation including the considered stress interaction terms and the mobility law is presented in this subsection for a better readability.

The model incorporates two coupled problems: The elastic problem calculating the stress field for a given plastic state and the dislocation problem describing the dislocation evolution for a given stress field yielding plastic deformation. Therefore, the distortion tensor Du is decomposed into an elastic part $\beta^{\text {el }}$ and a plastic part $\beta^{\mathrm{pl}}$ :

$$
\mathrm{Du}=\beta^{\mathrm{el}}+\beta^{\mathrm{pl}}
$$

Based on the assumption of small deformations, the strain $\varepsilon$ is given by the symmetric part: $\boldsymbol{\varepsilon}=\operatorname{sym} D \mathbf{u}$ and can be decomposed analogously into an elastic and plastic part: $\varepsilon^{\mathrm{el}}=\operatorname{sym} \beta^{\mathrm{el}}$ and $\varepsilon^{\mathrm{pl}}=\operatorname{sym} \beta^{\mathrm{pl}}$. The stress-strain relation describes physical linearity using the Cauchy stress tensor $\sigma=\mathbb{C}\left[\boldsymbol{\varepsilon}-\boldsymbol{\varepsilon}^{\mathrm{pl}}\right]$. Here $\mathbb{C}$ describes the elasticity tensor. The macroscopic equilibrium equation considering body forces $\boldsymbol{f}_{\mathcal{B}}$ is given in the continuum $\mathcal{B}$ by

$$
-\operatorname{div} \boldsymbol{\sigma}=\boldsymbol{f}_{\mathcal{B}} \quad \text { in } \mathcal{B}
$$

and complemented by the boundary conditions at the surface $\partial \mathcal{B}$ via given displacements $\boldsymbol{u}_{\mathrm{D}}$ for the displacements $\boldsymbol{u}$ on
Dirichlet boundaries $\partial_{\mathrm{D}} \mathcal{B}$ and given traction $\boldsymbol{t}_{\mathrm{N}}$ for the applied traction $\boldsymbol{\sigma} \boldsymbol{n}$ on Neumann boundaries $\partial_{\mathrm{N}} \mathcal{B}$. It holds

$$
\begin{array}{cc}
\boldsymbol{u}=\boldsymbol{u}_{\mathrm{D}} \text { on } \partial_{\mathrm{D}} \mathcal{B} & , \quad \boldsymbol{\sigma} \boldsymbol{n}=\boldsymbol{t}_{\mathrm{N}} \text { on } \partial_{\mathrm{N}} \mathcal{B} \\
\partial_{\mathrm{D}} \mathcal{B} \cup \partial_{\mathrm{N}} \mathcal{B}=\partial \mathcal{B} & , \quad \partial_{\mathrm{D}} \mathcal{B} \cap \partial_{\mathrm{N}} \mathcal{B}=\varnothing .
\end{array}
$$

Considering a slip system $s \in\{1, \ldots, S\}$, with the total number $S$ of considered slip systems (i.e. 12 in the fcc material), the single slip systems are characterized by their normal vector $\mathbf{m}_{s}$, their slip direction $\mathbf{d}_{s}=\frac{1}{b_{s}} \mathbf{b}_{s}$ and the line direction of positive edge dislocations $\mathbf{l}_{s}=\mathbf{m}_{s} \times \mathbf{d}_{s}$ forming an orthonormal basis $\left\{\mathbf{d}_{s}, \mathbf{l}_{s}, \mathbf{m}_{s}\right\}$. Herein, $\mathbf{b}_{s}$ is the Burgers vector of the length $b_{s}=$ $\left\|\mathbf{b}_{s}\right\|$. The plastic shear strains $\gamma_{s}$ on the individual slip systems, multiplied by the respective Schmid tensor $\mathbf{M}_{s}$, contribute to the plastic distortion

$$
\boldsymbol{\beta}^{\mathrm{pl}}=\sum_{s=1}^{S} \gamma_{s} \mathbf{M}_{s} \quad, \quad \mathbf{M}_{s}=\mathbf{m}_{s} \otimes \mathbf{d}_{s} .
$$

The elastic problem is closed by the evolution equation for the plastic shear strain in the dislocation problem via the Orowan's equation [23]

$$
\dot{\gamma}_{s}=v_{s} b_{s} \varrho_{s}
$$

based on the dislocation velocity $v_{s}$ and the total dislocation density $\varrho_{s}$ on the individual slip systems. The dislocation microstructure is described on each slip system by the following CDD quantities: The total dislocation densitiy $\varrho_{s}$, the vector of GND densitiy $\boldsymbol{\kappa}_{s}$, and the curvature density $q_{s}$. The vector of GND density can be written based on its screw and edge part: $\boldsymbol{\kappa}_{s}=\kappa_{s}^{\text {screw }} \mathbf{d}_{s}+\kappa_{s}^{\text {edge }} \mathbf{l}_{s}$. The evolution of these CDD quantities defining the averaged dislocation state is given as

$$
\begin{aligned}
& \dot{\varrho}_{s}=-\nabla \cdot\left(v_{s} \boldsymbol{\kappa}_{s}^{\perp}\right)+v_{s} q_{s} \quad \text { with } \quad \boldsymbol{\kappa}_{s}^{\perp}=\boldsymbol{\kappa}_{s} \times \mathbf{m}_{s} \\
& \dot{\boldsymbol{\kappa}}_{s}=\nabla \times\left(\varrho_{s} v_{s} \boldsymbol{m}_{s}\right) \\
& \dot{q}_{s}=-v_{s} \nabla \cdot\left(\frac{q_{s}}{\varrho_{s}} \boldsymbol{\kappa}_{s}^{\perp}\right)-\boldsymbol{A}_{s} \cdot \nabla^{2} v_{s}+\dot{q}_{s}^{p r o d} .
\end{aligned}
$$

Here, the curvature density evolution is extended by a production term $\dot{q}_{s}^{\text {prod }}$ that enables the model to increase the number of dislocation loops within the system. For $\dot{q}_{s}^{\text {prod }}=0$ the formulation is conservative. Using the closure assumptions introduced in [12], the dislocation alignment tensor $\boldsymbol{A}_{s}$ is given as

$$
\boldsymbol{A}_{s}=\frac{1}{2}\left(\left(\varrho_{s}+\left\|\boldsymbol{\kappa}_{s}\right\|\right) \frac{\boldsymbol{\kappa}_{s}}{\left\|\boldsymbol{\kappa}_{s}\right\|} \otimes \frac{\boldsymbol{\kappa}_{s}}{\left\|\boldsymbol{\kappa}_{s}\right\|}+\left(\varrho_{s}-\left\|\boldsymbol{\kappa}_{s}\right\|\right) \frac{\boldsymbol{\kappa}_{s}^{\perp}}{\left\|\boldsymbol{\kappa}_{s}^{\perp}\right\|} \otimes \frac{\boldsymbol{\kappa}_{s}^{\perp}}{\left\|\boldsymbol{\kappa}_{s}^{\perp}\right\|}\right) .
$$

The dislocation problem is closed by a constitutive law for the dislocation velocity. Depending on a material specific drag coefficient $B>0$, the effective stress $\tau_{s}^{\text {eff }}$ and the yield stress $\tau_{s}^{\mathrm{y}}$, the velocity is defined by

$$
v_{s}=\frac{b_{s}}{B} \operatorname{sgn}\left(\tau_{s}^{\mathrm{eff}}\right) \max \left\{0,\left|\tau_{s}^{\mathrm{eff}}\right|-\tau_{s}^{\mathrm{y}}\right\}, \quad \tau_{s}^{\mathrm{eff}}=\tau_{s}-\tau_{s}^{\mathrm{b}} .
$$

Here, the effective stress consists of the resolved shear stress on a slip system $\tau_{s}$ and the back stress $\tau_{s}^{\mathrm{b}}$ given by

$$
\tau_{s}=\sigma \cdot \boldsymbol{M}_{s} \quad, \quad \tau_{s}^{\mathrm{b}}=\frac{D \mu b_{s}}{\varrho_{s}} \nabla \cdot \boldsymbol{\kappa}_{s}^{\perp}, \quad \tau_{s}^{\mathrm{y}}=a \mu b_{s} \sqrt{\sum_{\tilde{s}=1}^{S} \varrho_{\tilde{s}}} .
$$


Here, the resolved shear stress is calculated by the projection of the stress tensor including dislocation eigenstresses on each slip system according to [30]. The back stress considers short-range dislocation stress interaction according to [9, 28] depending on a material parameter $D$ and the shear modulus $\mu$. Furthermore, the yield stress accounts for hardening due to the interaction with forest dislocations from other slip systems within the averaging volume. Here, we consider the classical Taylor yield stress including a material parameter $a>0$ and the shear modulus $\mu$. The variable $\tilde{s}$ is the index of summation over the individual slip systems. Following the findings in [33], this is assumed to be a reasonable approximation for the studies on high symmetry configurations as presented in the following in this paper.

\subsection{Source Model}

We introduce a three-dimensional dislocation source model based on the production rate of homogenized dislocation loop structures considering their relaxation potential. The source model extends the CDD evolution equations by a production term contributing to the curvature density evolution assuming that at least one source exists within all averaging volumes.

The dislocation sources on the individual slip systems are activated locally if the effective stress on the individual slip system exceeds the respective critical source stress $\tau_{s}^{\text {crit }}$. This critical source stress is chosen according to [11] as the maximum dislocation line tension of a Frank-Read source with the source length $l_{s}$. Since the information about the exact source length in the microstructure is missing in the continuum, the average source length within the averaging volume is assumed to scale with the average dislocation spacing given inversely proportional to the square root of $\varrho_{s}$ with a constant factor $c^{\mathrm{FR}}$. Handling vanishing dislocation densities, a reference value for the source length $l_{s}^{\text {ref }}$ is introduced to limit the source length and the corresponding critical source stress to a reasonable value with respect to the averaging volume size. The initiation of yielding depending on the specimen size were studied among others in [35, 24, 6, 39] and can be used to determine $l_{s}^{\text {ref }}$ referring to $\tau_{s}^{\text {crit }}$. It holds

$$
\tau_{s}^{\mathrm{crit}}=\frac{\mu b_{s}}{l_{s}}, l_{s}=\min \left\{l_{s}^{\mathrm{ref}}, c^{\mathrm{FR}} \frac{1}{\sqrt{\sum_{\tilde{s}=1}^{S} \varrho_{\tilde{s}}}}\right\}, \quad \mathrm{c}^{\mathrm{FR}}>0 .
$$

The basic idea is to consider the effective source activation and the resulting stress relaxation rather than the single bowout process. Therefore, the system relaxation resulting from the source activation is determined by taking into account the impact of the local dislocation density production within an averaging volume. The macroscopic ideal plastic material behavior is considered as an upper limit for the production rate in the system. In the following, the term "local" is referred to an averaging volume, whereas "global" is referred to the entire system considered.

Since the dislocation production rate is globally limited, a model based on local production rates have to predict the global effect as the sum of all local activities. However, the local production rates are not known a priori, thus a predictor-corrector approach is used in the following. The local production rates contribute to the globally expected plastic strain rate based on the global dislocation production $\dot{\varepsilon}_{\mathrm{gl}}^{\mathrm{pl} \text { exp }}$, which is limited by the norm of the global strain rate $\dot{\varepsilon}_{\mathrm{gl}}=\left\|\dot{\varepsilon}_{\mathrm{gl}}\right\|$. For a considered system with the volume $V$ we write

$$
\frac{1}{V} \iiint_{V} \dot{\varepsilon}^{\mathrm{pl} \mathrm{exp}} d V=\dot{\varepsilon}_{\mathrm{gl}}^{\mathrm{pl}, \exp } \leq \dot{\varepsilon}_{\mathrm{gl}}=\frac{1}{V} \iiint_{V} \dot{\varepsilon} d V .
$$

Here, the globally expected plastic strain rate due to the global overall dislocation production is calculated by the volumetric mean of the globally expected plastic strain rate due to the local dislocation production $\varepsilon^{\mathrm{pl} \text { exp }}$. The norm of the global strain rate is calculated analogously by the volumetric mean of the norm of the local strain rate $\dot{\varepsilon}$. The local strain rate $\dot{\varepsilon}$ calculated from the elastic problem can be written by its norm and a Tensor $\mathbf{M}^{\text {ext }}$ as

$$
\dot{\varepsilon}=\|\dot{\varepsilon}\|=\dot{\varepsilon} \cdot \mathbf{M}^{\text {ext }} \quad, \quad\left\|\mathbf{M}^{\text {ext }}\right\|=1 .
$$

The globally expected plastic strain rate due to the local dislocation production is calculated by combining equations (4) and (12) to

$$
\dot{\varepsilon}^{\mathrm{pl}, \exp }=\sum_{s=1}^{S}|\underbrace{\dot{\xi}_{s}^{\text {prod }} \gamma_{s}^{\exp }}_{\dot{\gamma}_{s}^{\exp }} \underbrace{s y m\left(\boldsymbol{M}_{s}\right) \cdot \boldsymbol{M}^{\text {ext }}}_{\text {Schmid factor }}|
$$

using the local dislocation density production rate $\dot{\xi}_{s}^{\text {prod }}$ and the globally expected plastic shear strain $\gamma_{s}^{\exp }$ per local source activation on the individual slip systems. The predicted local dislocation production density rate is determined locally by the ratio of the resulting stress increase over the critical source stress to the stress relaxation per single source activation $\Delta \tau_{s}^{\text {src }}$ as

$$
\dot{\xi}_{s}^{\text {prod }}=\frac{1}{V^{\text {src }}} * \begin{cases}\max \left\{\frac{\left|\dot{\tau}_{s}^{\text {exp }}\right|-\left|\dot{\mid}_{s}^{\text {relax }}\right|}{\Delta \tau_{s}^{\text {sc }}}, 0\right\} & ,\left|\tau_{s}^{\text {eff }}\right|>\tau_{s}^{\text {crit }} \\ 0 & ,|| \tau_{s}^{\text {eff }} \mid \leq \tau_{s}^{\text {crit }}\end{cases}
$$

Here, the resulting stress increase is the difference of the rate for the expected stress increase $\dot{\tau}_{s}^{\exp }$ and the stress relaxation $\dot{\tau}_{s}^{\text {relax }}$ of already existing dislocations within the averaging volume $V^{\text {src }}$. Since dislocation annihilation is neglected in the present formulation, the production rates are not allowed to become negative. Consequently, the dislocation sources remain locally inactive if the existing dislocation content within the averaging volume is sufficient to relax the local stress increase. The expected stress increase on the individual slip systems can be derived as

$$
\dot{\tau}_{s}^{\exp }=\mathbb{C}[\dot{\boldsymbol{\varepsilon}}] \cdot \boldsymbol{M}_{s} .
$$

The stress relaxation of already existing dislocations within the averaging volume depends on subsequent microstructure evolution and is at this point unknown. Therefore $\dot{\tau}_{s}^{\text {relax }}$ is approximated referring to the current dislocation microstructure by combining the Orowan's equation and Hooke's law as

$$
\dot{\tau}_{s}^{\text {relax }}=\mu v_{s} b_{s} \varrho_{s} .
$$


In an averaging volume, the relaxation stress contribution of one single, newly generated dislocation loop can be written as

$$
\Delta \tau_{s}^{\mathrm{src}}=\mu \frac{b_{s}}{h^{\mathrm{src}}}=\mathrm{const}
$$

using the height $h^{\text {src }}$ of a dislocation producing averaging volume.

The globally expected plastic shear strain per local source activation is calculated based on the mean sheared area $A_{s}^{\text {av }}$ per dislocation loop on each slip system as

$$
\gamma_{s}^{\exp }=\frac{A_{s}^{\mathrm{av}} b_{s}}{V}
$$

Simplifying, we assume that the mean area $A_{s}^{\text {av }}$ potentially be sheared by the expanding dislocation loop is approximately the cross section of the considered specimen when the dislocation loop leaves the continuum at the surfaces. The averaged cross section assumed for each slip system can be approximated by the system volume divided by the maximum distance between two slip planes of a slip system in the structure. Regarding different loading scenarios, it has to be taken into account that a maximum of only half of the cross section is sheared by a dislocation loop for torsion and bending due to the linear stress distribution. Thus, for a geometry and loading depending approximation of the mean area it can be formulated simplified:

$$
A_{s}^{\text {av }}=\mathrm{c}^{\text {load }} \frac{V}{\mathbf{x}^{\text {geom }} \cdot \tilde{\mathbf{m}}} \quad, \quad c^{\text {load }}= \begin{cases}0.5 & , \text { torsion, bending } \\ 1 \quad & \text { shear, tensile } \\ & \text { and compression. }\end{cases}
$$

Here, $\mathbf{x}^{\text {geom }}$ describes the approximated geometric measures, i.e. length in $\mathrm{x}$-direction, height in $\mathrm{y}$-direction and width in $\mathrm{z}$-direction for a rectangular envelope of the system. The vector $\tilde{\mathbf{m}}=\left(\left|m_{x}\right|,\left|m_{y}\right|,\left|m_{z}\right|\right)^{T}$ contains the components of the slip normal as absolute values and ensures positive contributions to the scalar product.

Considering the upper limit of the global production rate referring to equation (11), the predicted local production rates are adjusted to the physically reasonable production rates $\dot{\xi}_{s, c o r}^{\text {prod }}$ according to the predictor-corrector approach as

$$
\dot{\xi}_{s, c o r}^{\text {prod }}=\min \left\{1, \frac{\mathrm{c}^{\mathrm{geom}} \dot{\varepsilon}_{\mathrm{gl}}}{\dot{\varepsilon}_{\mathrm{gl}}^{\mathrm{pl}, \mathrm{exp}}}\right\} \dot{\xi}_{s}^{\text {prod }} \quad, \quad \mathrm{c}^{\mathrm{geom}}>0 .
$$

Here, the geometric correction factor $\mathrm{c}^{\text {geom }}$ takes into account the actual geometry of the cross section with respect to the volumetric mean value calculated in equation (11). In the following, we choose ceom $^{\text {geo }}=3 / 4$ referring to a circular cross section of a torsional system. For a detailed derivation see Appendix $\mathrm{B}$ Using the assumption of forming circular loops in the continuum, we can formulate the production rate of the curvature density considering the production of averaged closed loops as a multiple of $2 \pi$ orientations as

$$
\dot{q}_{s}^{\text {prod }}=2 \pi \dot{\xi}_{s, c o r}^{\text {prod }}
$$

The production of curvature density by the source model leads to expanding dislocation loops in the ongoing density evolution. This can be interpreted analogously to the discrete bow out to build a dislocation loop out of a Frank-Read source in DDD. However, the bow out process is neglected in the continuum. Thus, it has to be remarked, that the production time step has to be larger than the time step, that a dislocation bow out needs to form a dislocation loop. Since we want to focus on the dislocation density generation in the system, we neglect dislocation multiplication by dislocation reactions in the present study. However, the formulation can be extended by reaction and multiplication mechanisms straight forward, e.g. by including the model according to [34].

\subsection{Numerical Implementation}

The formulation has been implemented into a two-scale numerical framework. Based on the numerical formulation given in [31], we combine a finite element approach using tetrahedrons with linear ansatz functions to solve the elastic problem and an implicit Runge-Kutta discontinous Galerkin scheme with full upwind flux and constant tetragonal elements for the dislocation problem. Simplifying but found to be efficient, we apply the same mesh resolution for both scales. The time discretization is realized by an implicit midpoint rule with a fixed time step.

Regarding the boundary conditions, we assume that the dislocations can leave the continuum unhindered on the open boundaries with the surface normal $\boldsymbol{n}$. This is described by Robin-type boundary conditions for the dislocation densities and a dissipation of the curvature density on the outflow boundaries $\partial_{\text {out }} \mathcal{B}$. Furthermore, no dislocations are allowed to enter the system over the surface. This means that the curvature density on the inflow boundaries $\partial_{\text {in }} \mathcal{B}$ vanishes. Thus, it holds

$$
v_{s}\left(\left\|\boldsymbol{n} \times \boldsymbol{m}_{s}\right\| \varrho_{s}+\left(\boldsymbol{n} \times \boldsymbol{m}_{s}\right) \cdot \boldsymbol{\kappa}_{s}\right)=0 \text { on } \partial \mathcal{B}
$$

and

$$
\begin{gathered}
q_{s}=0 \text { on } \partial_{\text {in }} \mathcal{B}, \quad \partial_{\text {in }} \mathcal{B}=\left\{\mathbf{x} \in \partial \mathcal{B}: \frac{\nu_{s}}{\varrho_{s}} \cdot \boldsymbol{\kappa}_{s}^{\perp}<0\right\} \\
\partial_{\text {in }} \mathcal{B} \cup \partial_{\text {out }} \mathcal{B}=\partial \mathcal{B}, \quad \partial_{\text {in }} \mathcal{B} \cap \partial_{\text {out }} \mathcal{B}=\varnothing .
\end{gathered}
$$

\subsection{Considered system}

To study the characteristics of the evolving microstructure in face-centered cubic microwires under torsion the following system configuration is considered. We investigate a single crystalline microwire with a length of $48 \mu \mathrm{m}$ and a radius of $R=12 \mu \mathrm{m}$. The bottom and the top of the wire are subjected to symmetric torsional loading. The system as well as the chosen discretization is shown in Fig. 11 Regarding the boundary conditions, there is no restriction for the nodal displacement in length direction of the wire. Only the center node at the bottom is fixed to prevent rigid body motion. The circumference surfaces are considered traction free.

Mimicking aluminum, the material is assumed to be isotropic with a $\langle 100\rangle$ crystal orientation characterized by the shear modulus $\mu$ and the Poisson's ratio $v$ given in Fig. 1 . 

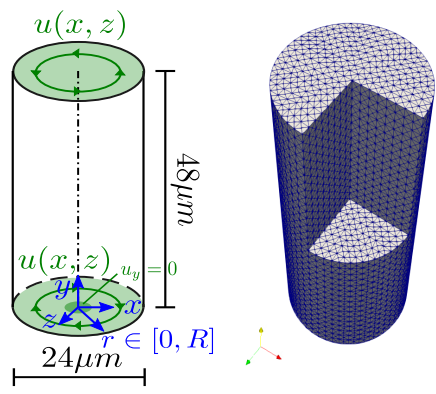

$\begin{array}{rll}\mu & =27 & \mathrm{GPa} \\ v & =0.347 & \\ b & =0.286 & \mathrm{~nm} \\ B & =10^{-4} & \mathrm{~ns} \mathrm{GPa} \\ D & =0.255 & \\ a & =0.35 & \\ l_{s}^{\text {ref }} & =0.22 & \mu \mathrm{m} \\ c^{\mathrm{FR}} & =1.5 & \\ c^{\mathrm{geom}} & =0.75 & \end{array}$

Fig. 1: Geometry, finite element mesh (170 k elements), and material parameters for the microwire under torsion loading.

The length of the Burgers vector $b$, a material specific drag coefficient $B$ [20], the backstress parameter $D$ [28], as well as the material parameter for the yield stress $a$ [19] are chosen according to the respective references. For the source model, the reference source length $l_{s}^{\text {ref }}[32]$, the constant $c^{\mathrm{FR}}$ (chosen according to [29]), and the geometric correction factor for the production density $c^{\text {geom }}$ are also given in Fig. 1 .

Based on the loading and the crystal orientation, we want to analyze the dislocation density evolution on the fcc slip systems. Fig. 2 shows theoretical considerations of the interplay of the different slip systems with respect to the applied shear stress. Looking at the different system orientations, the structure (see Fig. 2(a)) can be relaxed in two different ways: In the simplified system, the plastic deformation takes place on a plane parallel to the load plane yielding the deformation by plastic slip on this plane, see $\beta_{21}^{\mathrm{pl}}$ and $\beta_{21}^{\mathrm{el}}$ in Fig. 2(b). Or, the plastic deformation can take place perpendicular to the load plane creating an elastic counterpart to the elastic shear loading, see $\beta_{12}^{\text {el }}$ and $\beta_{21}^{\text {el }}$ in Fig. 2(c). Since the strain tensor is calculated by the symmetric part of the distortion tensor, the two transposed components cancel out.

Due to these relaxation principles, the interaction of the different slip systems can be identified as visualized in Fig. 3 for torsional loading. Using a cartesian coordinate system, the re- sulting stress tensor due to torsion consist of two shear stress components, here $\tau_{x y}$ and $\tau_{y z}$, with a linear distribution over the structure. Simplifying, slip systems with different slip planes but same Burgers vector can be combined into one effective slip system producing the same plastic distortion according to equation 4. It can be found, that there are two ways (Fig. 3 (a) and (b): $\mathrm{A} 6+\mathrm{B} 5+\mathrm{C} 5+\mathrm{D} 6$ and $\mathrm{A} 2+\mathrm{B} 2+\mathrm{C} 1+\mathrm{D} 1)$ to generate a counter part for the relaxation (cp. Fig. 2(c)) seeing one torsional stress component. The resulting Burgers vector points into the direction of the torsion axis and the slip plane normal is orthogonal to the torsion axis. Furthermore, there are two slip system combinations (Fig. 3(c) and (d): B4+D4 and A3+C3) showing a resulting slip plane normal directed in the torsion axis, transforming the elastic into plastic deformation (cp. Fig. 2.b)) and seeing both torsional stress components. Here, the neutral axis is rotated by $45^{\circ}$.

Considering the resulting options for plastic relaxation, a simplified set of four reduced effective slip systems is examined. Considering the possible dislocation density evolution on the reduced effective slip systems leads to a distribution of pure screw GNDs with opposite sign on slip planes perpendicular (Fig. 3.c) and (d): B4+D4 and A3+C3) and parallel (Fig. 3.a) and (b): $\mathrm{A} 6+\mathrm{B} 5+\mathrm{C} 5+\mathrm{D} 6$ and $\mathrm{A} 2+\mathrm{B} 2+\mathrm{C} 1+\mathrm{D} 1)$ to the torsion axis due to the linear torsional stress field and the resulting pileups. This is consistent to the theoretical considerations for the analysis of twist boundaries given in [36]. Although we analyze all twelve slip systems for the microwire in the following, the considerations of effective slip systems give a theoretical understanding of possible relaxation mechanisms in the system.

The plastic distortion resulting from the interplay of all active slip systems leads to a misorientation of the cross section with respect to the rotation axis as shown in Fig. 4. For the derivation of the misorientation from the distortion fields see Appendix D. It can be observed, that the rotation axes point towards the wire center for an activation of all four slip systems with the Burgers vector orthogonal to the torsion axis $(\mathrm{A} 3+\mathrm{B} 4+\mathrm{C} 3+\mathrm{D} 4)$. However, looking at the local contribution of specific slip systems, it can be seen that this leads to a uniform orientation of the rotation axis parallel to the torsion axis over the cross section. (a)

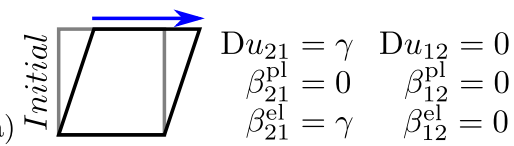

(b)

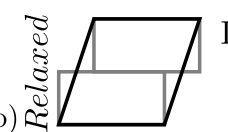

$\begin{array}{rrr}\mathrm{D} u_{21}=\gamma & \mathrm{D} u_{12}=0 \\ \beta_{21}^{\mathrm{pl}}=\gamma & \beta_{12}^{\mathrm{pl}}=0 \\ \beta_{21}^{\mathrm{el}}=0 & \beta_{12}^{\mathrm{el}}=0\end{array}$

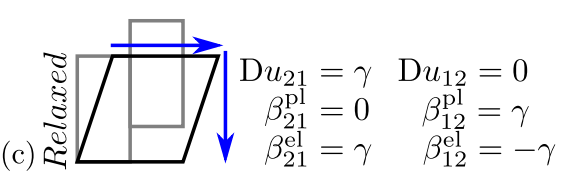

Fig. 2: Two possible relaxation principle are shown $((a) \rightarrow(b)$ and $(a) \rightarrow(c))$, whereby the deformed structure is drawn in black, the plastic deformation is shown in gray and the elastic deformation is represented by blue arrows.

(a)

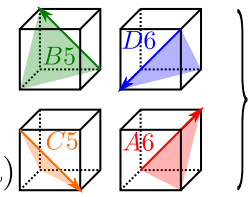

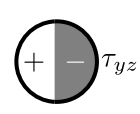

(b)

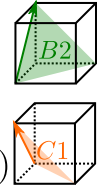

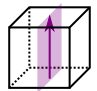

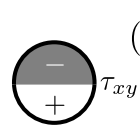

(d)

(c)

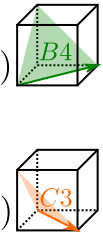

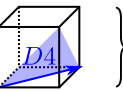
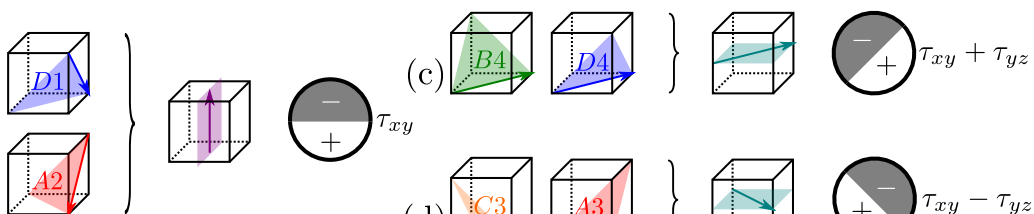

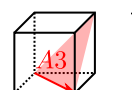

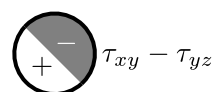

Fig. 3: The resulting plastic deformation, see eq. 4, through the combination of different slip systems and the relevant external stress components for the dislocation velocity, see $\tau_{s}$ in eq. 90 are shown for a $\langle 100\rangle$ crystal orientation and torsional loading. 

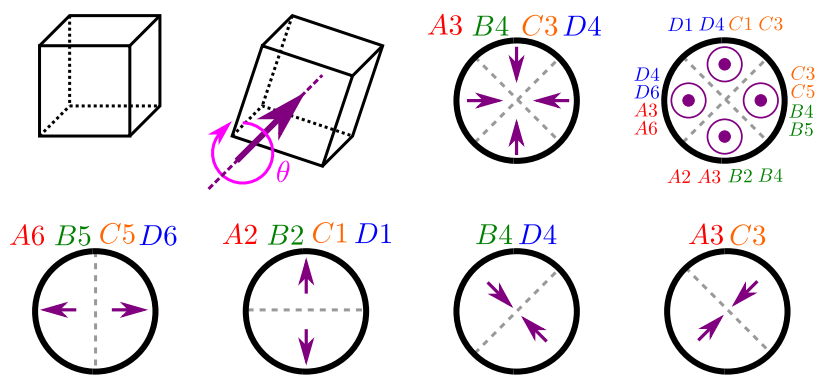

Fig. 4: Resulting torsion axes for the interplay of plastic slip on specific slip systems. The misorientation is characterized by the rotation axis (purple arrow) and the rotation angle $\theta$. Considering only the skew symmetric part of the plastic distortion, the slip systems distortion can cancel out.

\section{Results}

We study the system of a microwire under torsional load according to 2.4 The distributions over a cross section perpendicular to the torsion axis shown in the following are evaluated at half of the wire length. Curves over the (normalized) radius are computed by averaging over the circumference for a given radius at half of the wire length.

\subsection{Benchmark system}

In order to test the introduced source model, a simple benchmark system is considered. The torsional load is given by a wire distortion of $2.4^{\circ}$ and the slip systems are examined separately considering one slip system at a time. For simplicity, we allow for the dislocation production in one single averaging volumes only and track the dislocation density evolution out of a single source activation as shown in Fig. 5 for the slip system $B 4$ and in Fig. 6 for $B 5$. The produced curvature density leads to an expanding dislocation loop, leaving the continuum at the surface and piling-up in the middle of the wire according to the neutral axis of the applied stress field. With respect to the sign of the stress field, the curvature density resulting from a source activation can be identified as positive or negative curvature density. However, the GND pile-ups at the neutral axis can still consist of e.g. negative screw density on both sides of the neutral axis as e.g. for slip system B4.

\subsection{Relaxation of a constant torsional load}

We consider the microwire according to Sec 2.4 and apply a constant torsional deformation of $1^{\circ}$ to be relaxed. Here, the full fcc system is considered with a homogeneous distribution of sources. Due to the linear stress field, the dislocation density is produced near the circumferential surface. While a significant part of the produced density leaves the system over the
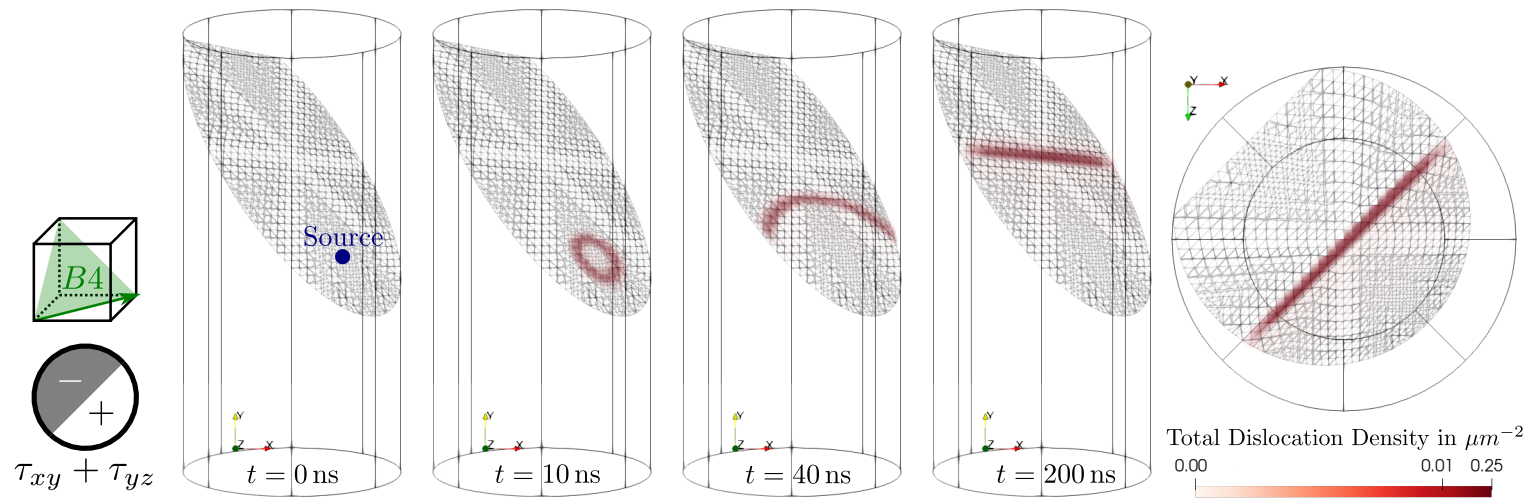

Fig. 5: Benchmark system with a single source activation and the evolution of the total dislocation density of the slip system B4. The top view of the cross section shows the converged GND density consisting of nearly $100 \%$ screw density close to the neutral axis.
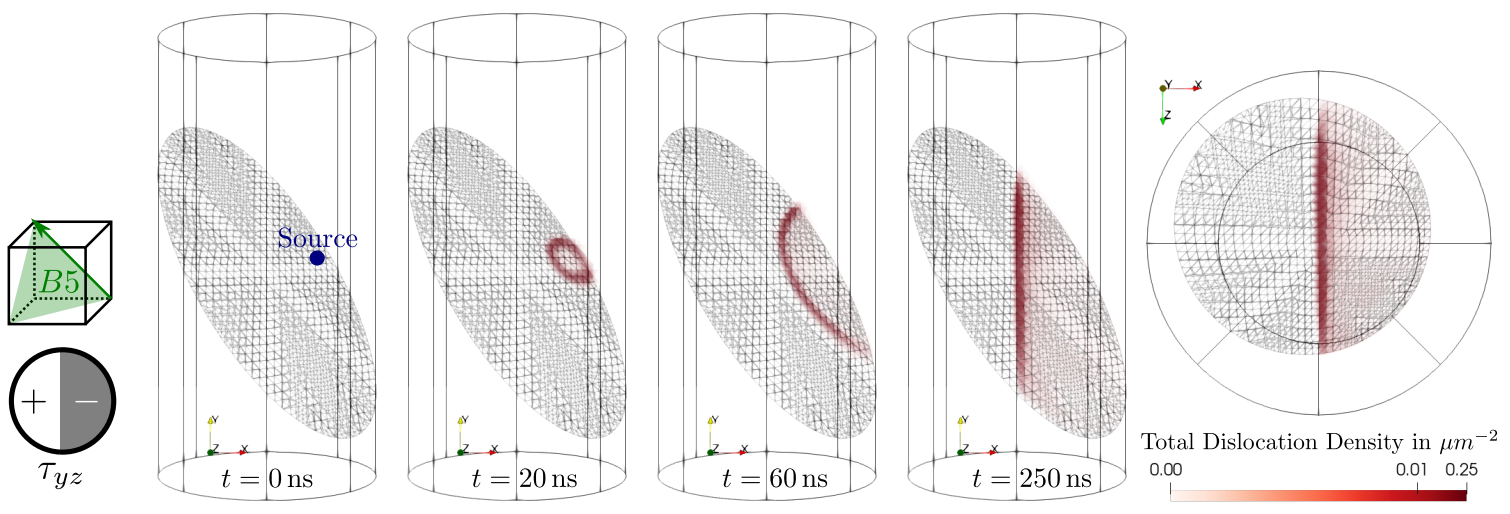

Fig. 6: Evolution of the total dislocation density of the slip system B5 for the benchmark system. The nearly converged GND density consist of $34 \%$ screw part and $66 \%$ edge part. 

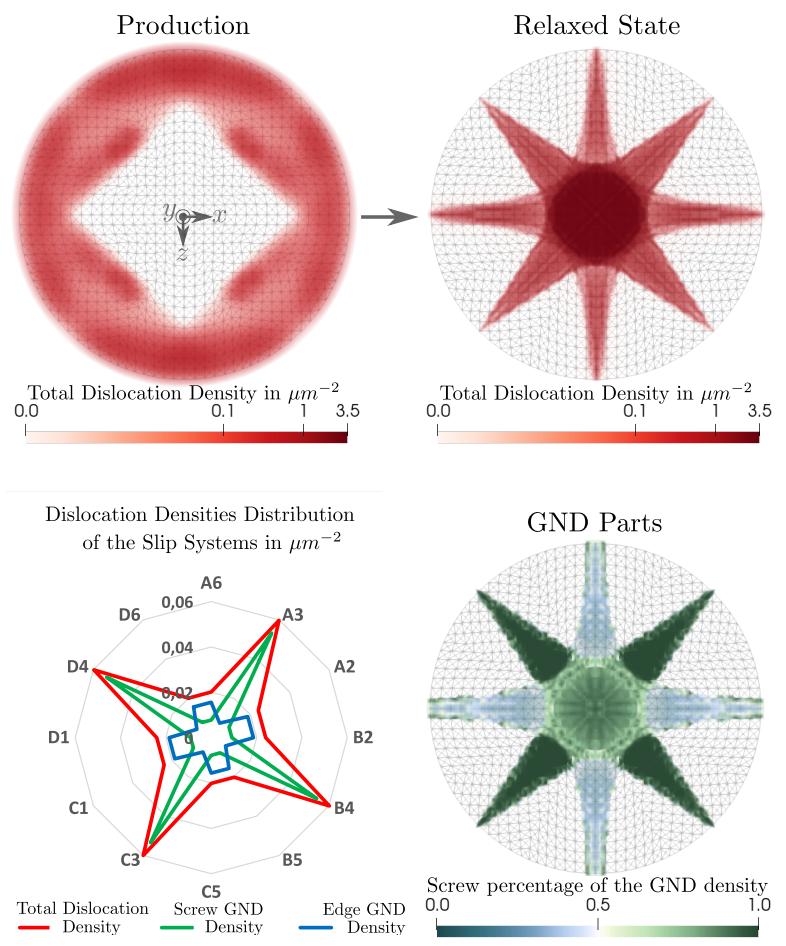

Fig. 7: Spatial distribution of the total dislocation densities $\varrho$ at an early stage of the density production (top left) and in the relaxed state (top right) as well as the ratio of the slip systems contributions (bottom left) and the screw and edge part of the GND density (bottom right) for the relaxed state.

surface (cp. the evolution of a single loop in the Benchmark system), the evolving dislocation density in the system forms an inhomogeneous, star-like spatial distribution over the cross section. Due to the applied stress fields there occur dislocation pile-ups at the neutral axes of the slip systems, respectively. The density consists mainly of screw GNDs from the four slip systems with the Burgers vector orthogonal to the torsion axis as shown in Fig. 7 Measuring the density production rate for the different slip systems, the production rate of the slip systems with a Burgers vector orthogonal to the torsion axis is found to be three times higher compared to the other systems. The resulting total dislocation density distribution in the cross section for a crystal orientation rotated by $22.5^{\circ}$ along the torsion axis can be found in Fig. E.14 in Appendix E

The spatial distribution of the dislocation densities, summed up over all slip systems $\left(\varrho=\sum_{\tilde{s}=1}^{S} \varrho_{\tilde{s}}, \kappa^{\text {screw }}\right.$ and $\kappa^{\text {edge }}$ are calculated analogously), and the strain components over the radius are shown in Fig. 8 for the relaxed state. Here, the total dislocation density - consisting mainly of GNDs (about $74 \%$ screw GNDs and $26 \%$ edge GNDs) - pile up in the middle of the wire forming a plateau-like distribution with $\varrho \approx 3 \mu \mathrm{m}^{-2}$. The results of the screw GND density can be compared to the density predicted by the Critical Thickness Theory (CTT) according to [18]. The computed density in the center of the wire shows similar results as the theoretically predicted CTT density, that takes into account only screw GNDs and considers a surface shear $\gamma_{R}$
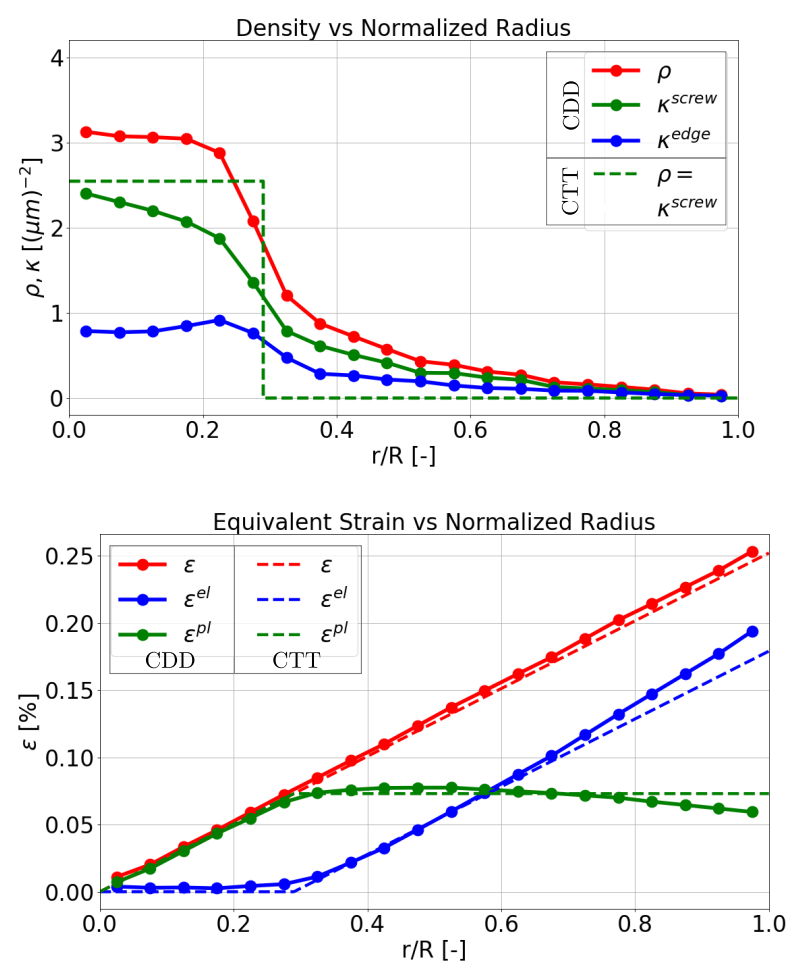

Fig. 8: Spatial distribution of the dislocation densities and strain components over the radius for the relaxed state compared to the CTT-prediction shown by dashed lines.

as: $\kappa_{\mathrm{CTT}}^{\text {screw }}=2 \frac{\gamma_{R}}{R b}$. Evaluating the equivalent total strain in the system, a linear distribution over the radius from $\left.\varepsilon_{\text {eq }}\right|_{r=0}=0$ up to $\left.\varepsilon_{\mathrm{eq}}\right|_{r=R}=0.25 \%$ is observed that consists of a plastic part in the inner region of the wire until a transition point at about $0.3 \frac{r}{R}$. We call the corresponding radius of this transition point critical radius $r^{\text {crit }}$ in the following. Outside this transition point, the gradient of the plastic strain is negligible, whereas the elastic part increases linearly.

We denote the torsion angle at which plastic yielding starts as critical torsion angle $\varphi^{\text {crit }}$. A comparison of the radial distribution of the strain and the GND density with CTT for multiples of $\varphi^{\text {crit }}$ is shown in Fig. 9
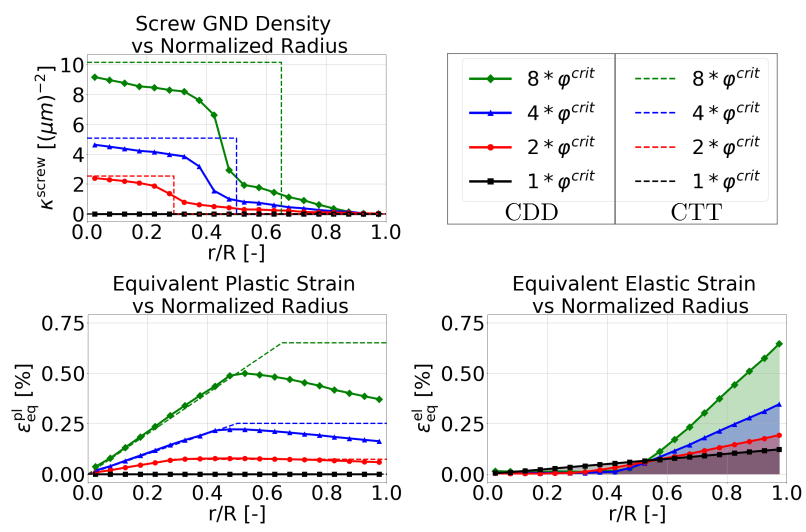

Fig. 9: Radial distribution of strain and GND densities components compared to the CTT for different torsion angles. 
The results show, that the transition points of the plastic and elastic zones in CDD correspond to CTT for smaller loads and start varying for higher loading. However, the integral of the elastic strain over the radius (cp. the colored areas in Fig. 9) is assumed to be constant in CTT once the critical torsion angle is exceeded. This holds only approximately true for the CDD results. For an applied torsion angle that is $N$ times the critcial torsion angle, the CTT predicts a critical radius of $r_{\mathrm{CTT}}^{\text {crit }}=(1-$ $\left.\frac{1}{\sqrt{N}}\right) R$. Accordingly, the critical radius for $N \in\{2,4,8\}$ is $r_{\text {CTT }}^{\text {crit }}=$ $\{0.29,0.5,0.65\} R$. Similar values are derived for the critical radius within the CDD computations. These are approximately $\{0.28,0.43,0.48\} R$.

\subsection{Diameter variation of the microwire}

To analyze the influence of the microwire diameter on the pile-up, we study the system of Sec. 3.2 with a radius varied to $\{48,12,6,3\} \mu m$. The systems are considered to have the same aspect ratio of wire radius to wire length and all of them are loaded with the same torsion angle of $\varphi=1^{\circ}$. Consequently, the surface strain $\gamma_{R}$ is identical for all micowires but the strain gradient increases for smaller microwire radii. We mesh each system with the same number of elements leading to the same resolution along the normalized radius $\frac{r}{R}$. However, this implies a different resolution along the coordinate $r$. This might have slight effects on the distribution, but we assume that these effects are negligible due to the considered backstress term that corrects the near field of the unresolved mean field stresses ac-

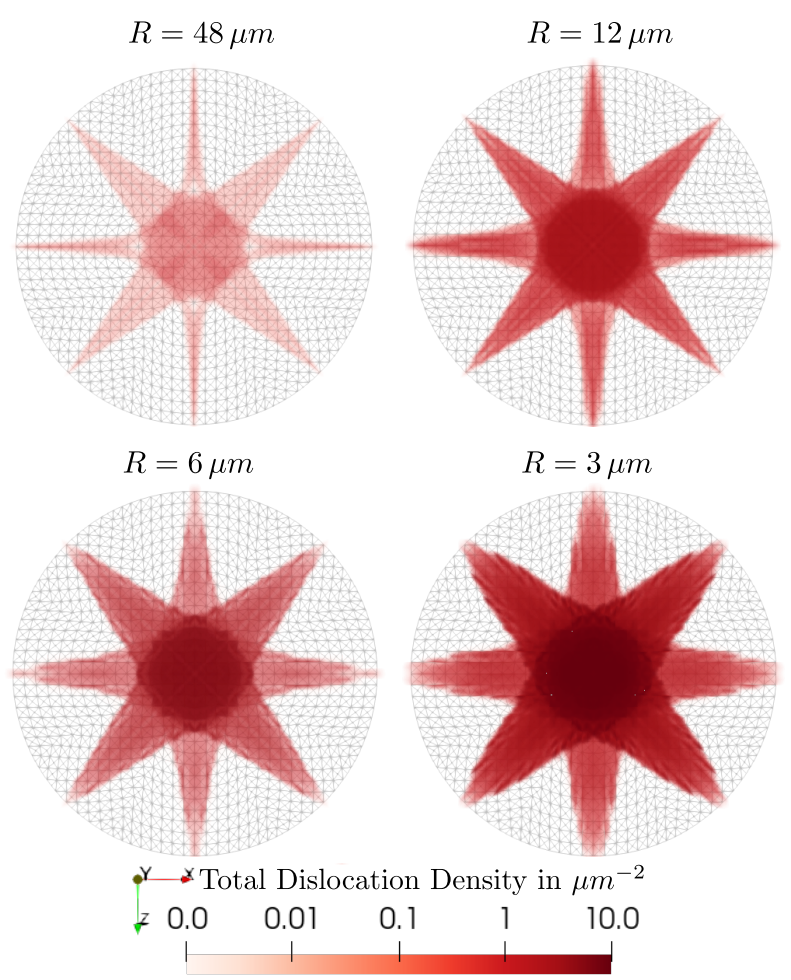

Fig. 10: Spatial distribution of the total dislocation densities $\varrho$ in the microwire cross section at the relaxed state for microwires with different radii $R$ under constant torsional load $\left(\varphi=1^{\circ}\right)$. cording to [28]. The reference value for the source length referring to the critical stress for the source activation and therefore the start of the yielding is an input variable of the continuum source model and chosen constant in this study. The impact of varying $l_{s}^{\text {ref }}$ according to experimental findings for different microwire sizes on the initiation of yielding is shown in Fig. F.15 in Appendix F.

The relaxed dislocation microstructures in the respective cross section at half of the microwire length are shown for the different wire radii in Fig. 10 For all microwire radii, the star-like distribution of the total dislocation density is observed. However, the amount of density involved increases for smaller microwire radii. Furthermore, the length of the dislocation pileup with respect to the normalized radius increases for smaller wire radii leading to a broadening of the star-like density formation.

The spatial distributions of the total dislocation density and the equivalent plastic strain over the normalized radius are shown in Fig. 11. For comparability, the dislocation density curves for different microwire radii are normalized with respect to $\kappa_{\text {norm }}^{\text {screw }}=1.93 \frac{\gamma_{R}}{R b}$ according to the experimental findings for fcc polycrystals under torsion by [1].

Here, similar distributions are observed for wires with a radius of $R=12 \mu \mathrm{m}$ and $R=48 \mu \mathrm{m}$, whereas the results for micorwires with smaller radii show lower normalized dislocation densities and smaller equivalent plastic strain gradients near the center. The resulting normalized torque is measured based on the stress distribution in the cross section according to Appendix C as $\frac{M_{T}}{R^{3}}=123 \mathrm{MPa}$ for $R=48 \mu \mathrm{m}$ and $\frac{M_{T}}{R^{3}}=134 \mathrm{MPa}$ for $R=3 \mu \mathrm{m}$.

Normalized Dislocation Density vs Normalized Radius
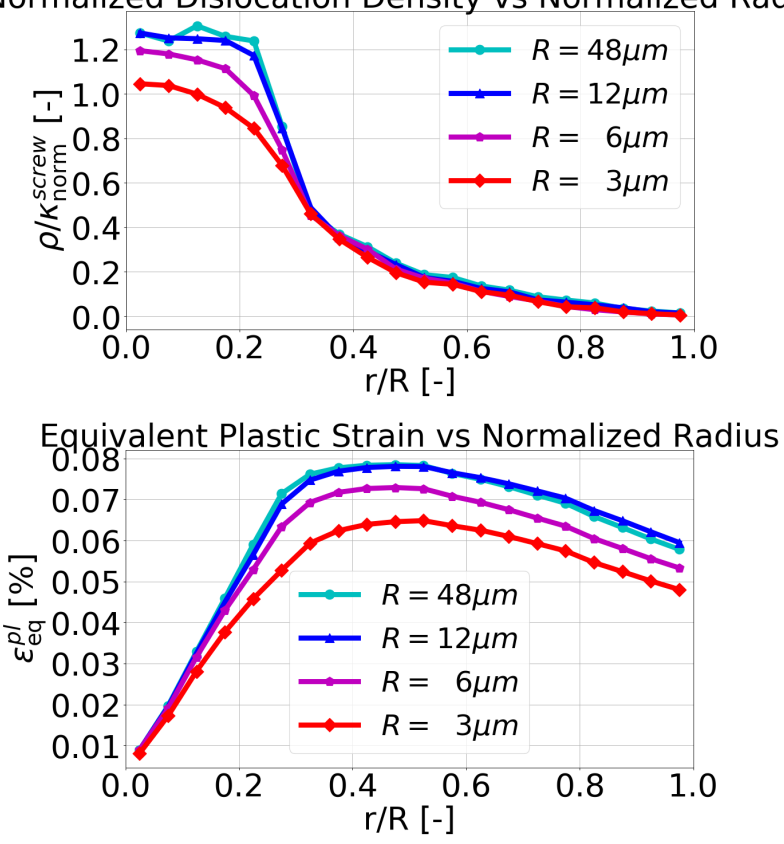

Fig. 11: Normalized total dislocation density and equivalent plastic strain over the normalized radius for micorwires with different radii $R$ under constant torsional $\operatorname{load}\left(\varphi=1^{\circ}\right)$. 


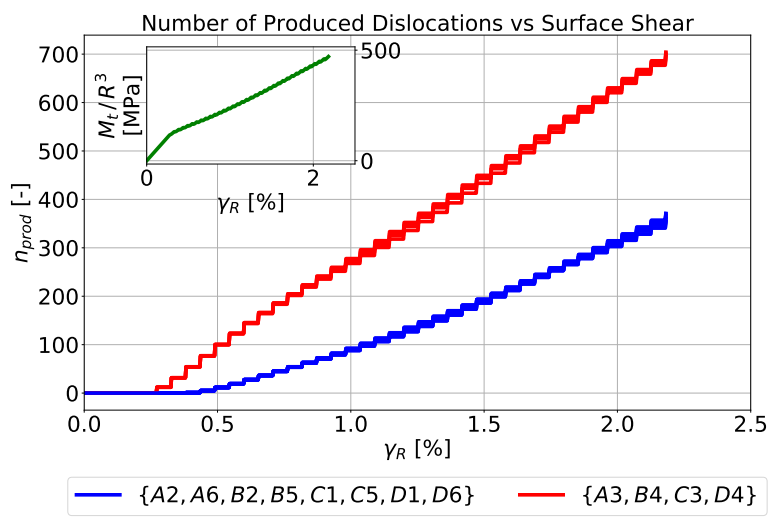

Fig. 12: Normalized torque and number of produced dislocation loops depicted over the surface shear for a constant torsional rate of $0.5 \frac{\circ}{\mu s}$.

\subsection{Constant torsional rate}

To compare the formulation with experimental data from the literature, the system (diameter of $24 \mu \mathrm{m}$ ) is subjected to a constant strain rate $\left(+0.5 \frac{\circ}{\mu s}\right)$ up to a torsion angle of $\varphi=5^{\circ}$. During the loading, it can be observed, that the slip systems with the Burgers vector oriented orthogonal to the torsion axis are activated earlier than the others. However, finally all slip systems are activated and show a dislocation density evolution according to the high symmetry orientation, see Fig. 12. After the elastic-plastic transition, the increase of the total amount of produced dislocation density corresponding to the number of produced dislocation loops is found to be almost linear.

Considering the local misorientation, the orientation of the continuum in the neutral axis with respect to its initial configuration as well as the misorientation over the cross sections along the length of the wire with respect to the respective current averaging volume in the neutral axis are shown in Fig. 13. The simulation results are derived for a plastic equivalent strain at the surface of $\left.\varepsilon_{\mathrm{eq}}^{\mathrm{pl}}\right|_{r=R}=0.2 \%\left(\varphi=2^{\circ}\right)$ and compared to experimental tests according to [43]. The experimental results show two normalized misorientation maps from cross sections located in the middle of the respective $\langle 100\rangle$ oriented grain of different twisted bamboo-structured Au microwire specimens with a diameter of $25 \mu \mathrm{m}$ for a plastic surface shear of $\gamma_{R}^{\mathrm{pl}}=0.4 \%$ (corresponding to $\left.\varepsilon_{\mathrm{eq}}^{\mathrm{pl}}\right|_{r=R}=0.23 \%$ ). Although measured under the same conditions, the two experimental results differ qualitatively. The left experimental result in Fig. 13 shows the highest gradients of the misorientation angle near the wire center, whereas the misorientation angle for the right experimental result vanishes near the wire center and the highest gradients of the misorientation angle can be found near the surface. Further details on the experimental setup are given in [43].

Considering the simulation results for a plastic equivalent strain at the surface of $\left.\varepsilon_{\mathrm{eq}}^{\mathrm{pl}}\right|_{r=R}=0.2 \%\left(\varphi=2^{\circ}\right)$, the rotation axes in the cross section at half of the wire length are pointing towards the center and the highest gradients of the misorientation angle $\theta$ occur near the wire center. In contrast, the rotation axes align to the torsion axis at the top and the bottom of the
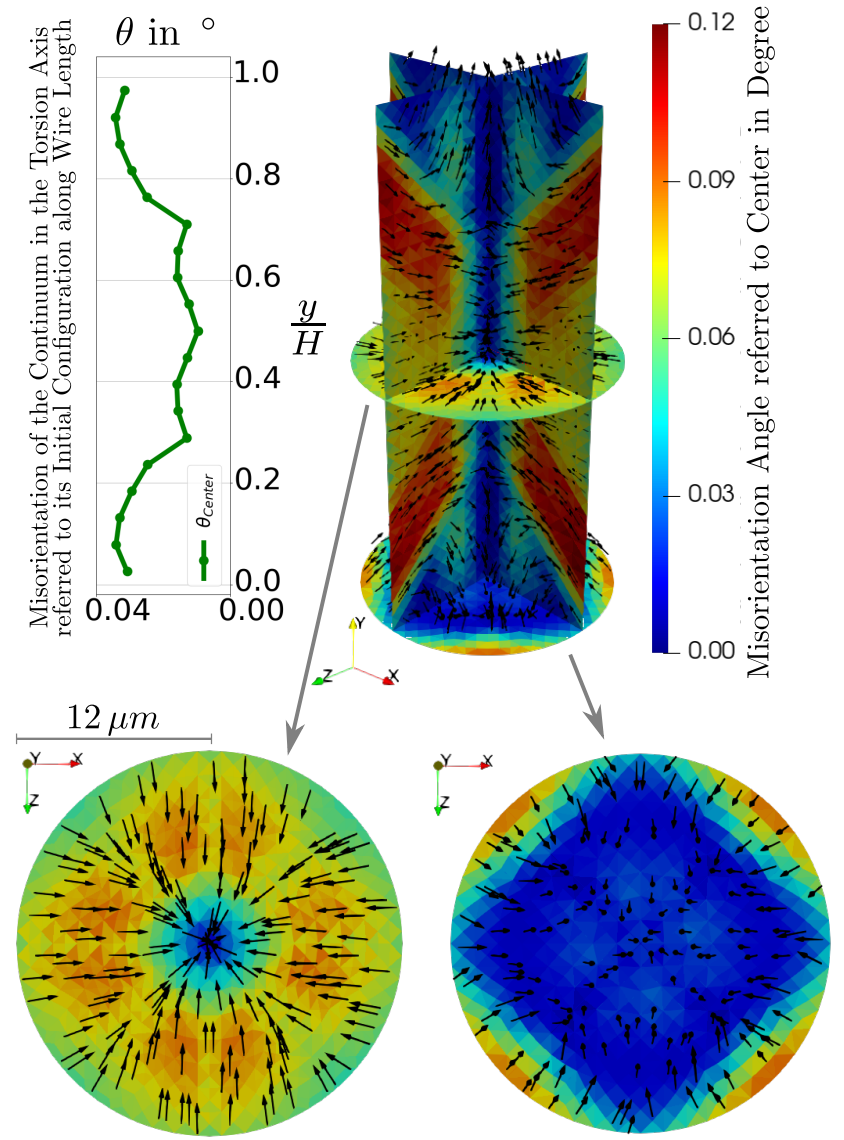

Simulation

Experiments

Normalized Misorientation Angle referred to Center

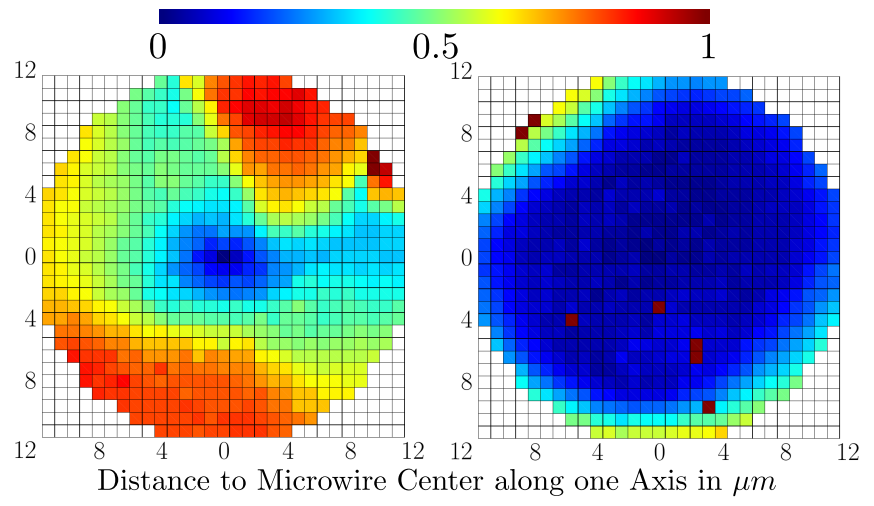

Fig. 13: Simulation results for the misorientation of the continuum in the torsion axis referred to the initial configuration along the length of the wire (green curve) and misorientation distribution for different cross sections referred to the respective wire center, whereby the rotation angle $\theta$ is shown in color and the rotation axis $\mathbf{n}^{\text {rot }}$ by black arrows. For comparison, experimental results are shown for the misorientation referred to the respective wire center normalized to the maximal occurring misorientation angle in the cross section. 
wire, shown for the bottom cross section in Fig. 13 Here, the highest gradients of $\theta$ can be found near the surface. Furthermore, it can be found in the simulation that the distributions of the misorientation angle over the respective cross section show four axis symmetries.

Tracking the torsion axis from the bottom to the top surface, it can be observed that the wire center is rotated by about $0.034^{\circ}$ compared to the initial configuration for the bottom and the top cross sections. However, the center point of the cross section at half of the wire length has almost no misorientation compared to its initial orientation showing a rotation angle of $0.01^{\circ}$.

\section{Discussion}

For the analysis of the plastic behavior and the underlying dislocation evolution of microwires under torsion, a profound understanding for the relaxation mechanisms and dislocation behavior on the single slip systems is essential. Therefore, the presented dislocation based continuum formulation incorporating a dislocation source model has been applied to single crystalline microwires with $\langle 100\rangle$ crystal orientation.

It is observed that the slip systems can be divided into two groups according to the crystal orientation considering the relaxation principle (see Fig. 2 and Fig. 3) and the ratio of screw and edge GND densities in the dislocation pile-ups (see Fig. 5 and Fig. 6). The dislocation density pile-ups in the slip systems with a Burgers vector orthogonal to the torsion axis (A3, B4, C3 and D4) consists almost entirely of screw density whereas for the pile-ups of the other slip systems, the major part consists of edge density. Thus, the total screw-edge composition of the GND density is directly linked to the ratio of the amounts of dislocation density on the different slip systems (see the GND parts in Fig. 7 with respect to the distribution of the piled up dislocation densities in Fig. 5 and Fig. 6.

Since the slip systems with the Burgers vector orthogonal to the torsion axis are affected by both torsional shear stress components, these slip systems are favored. The results show, that this can be observed by the higher production rate for the relaxation problem and the earlier activation for the constant torsional rate. Therefore, the GND density consists for small torsional angles mainly of screw denstiy. However, in contrast to a simple shear or tensile problem, there results a linear stress distribution in the continuum due to the torsion loading. The maximum stresses occurring in the system increase even if the global strain increase is relaxed completely by plastic slip (cp. the maximum of the elastic strains in Fig. 9p. Consequently, the slip systems with a Burgers vector not orthogonal to the torsion axis are also activated with increasing torsion angle and the composition of screw and edge parts changes as well as further quantities, like the spatial distribution of the total dislocation density or orientation of the rotation axes for the misorientation.

Due to the torsional loading, the highest stresses occur near the surface and activate the dislocation source model in this region according to the introduced formulation. The productionfree area at the beginning of the density evolution, showing a square shape in the cross section (see Fig. 7), is confined by the internal limit, at which the critical stress is reached. The boundary line of production-free and production area is parallel to the neutral axes of the individual slip systems and is also found by molecular dynamics simulation of twisted, $\langle 100\rangle$ oriented Au nanowires [37]. Although the governing source mechanisms can differ with varying sample size e.g. due to the activation of Frank-Read sources, truncated sources, dislocation nucleation from the surface, or the 'starvation' of mobile dislocation density, the continuum representation focus on dislocation sources mechanisms corresponding to Frank-Read sources to formulate an averaged model bridging from DDD to CDD representation. According to the stress field, the dislocations move into the wire and pile up at the neutral axes of the individual slip systems. Therefore, the location of dislocation production and accumulation of dislocation density differs on each individual slip system for the considered case. Consequently, the critical stress for the source activation does not change during the simulation for the chosen system since the source length calculated by the total dislocation density at the production areas does not exceed the reference source length.

By the combination of the different slip systems and their rotated velocity field, a star-like dislocation density distribution results. The star-like distribution of dislocations is also found by a discrete dislocation dynamics simulation of $1 \mu \mathrm{m}$ thick copper micorwires under torsion for a $\langle 100\rangle$ crystal orientation in [26] as shown in Fig. G.16 in Appendix G According to the dislocation pile-ups at the respective neutral axes on the individual slip systems, a stable dislocation density distribution occurs not only in the specimen center, but also near the specimen surface as shown in Fig. 7. This is in contrast to the critical thickness theory according to [18] and the results of the continuum dislocation approaches in [13] and [14] assuming artificial, axially symmetric slip systems. The star-like configuration is directly linked to the crystal orientation as shown in Fig. E.14 in Appendix Efor a crystal orientation rotated around the torsion axis by $22.5^{\circ}$.

For the relaxed state, a nearly constant total dislocation density can be observed near the center, which is also found by continuum dislocation approaches in [13, 14]. Thereby, the amount of screw GND density in the center for a $24 \mu \mathrm{m}$ thick microwire is consistent with the experimental findings for fcc polycrystals under torsion by [1] quantifying the Nye's factor (ratio of GND density multiplied with Burgers vector length to the plastic strain gradient) to 1.93 . Thus, the empirical calculation can be used for an estimation of the screw dislocation density in the microwire center also for single crystals, if the external stresses dominate over the internal stresses.

The dislocation density produced near the surface moves towards the center causing plastic slip and relaxing the continuum. Consequently, the material near the torsion axis is plastically deformed although the external stress is lower than the critical source stress. It is remarked, that this is an important feature of the nonlocal continuum formulation since such a behavior can not be captured by local continuum approaches without flux terms.

Due to the stress induced motion of dislocations, it is found that the radial location of the drop of the total dislocation den- 
sity and the transition point towards an increasing elastic strain correlates as shown in Fig. 8. The location of this transition point is consistent to the CTT for small torsion angles [18]. However, the transition of the dislocation densities in the pileups is rather smooth compared to the sharp transition as predicted by CTT due to the internal stresses.

For the dislocation microstructure, the dislocation density in the pile-ups is balanced between the external stress field moving the dislocation density towards the respective neutral axes of the slip systems and the mean field stresses or backstresses of the GND density. Thus, the proportion of external and internal stress contributions plays an important role for the dislocation distribution and the associated distribution of the plastic slip in the pile-ups. This yields size effects with respect to a constant torsional load as shown in Fig. 10 and Fig. 11. The increasing amount of dislocation density for smaller microwire radii $\left(\kappa_{\text {norm }}^{\text {screw }} \propto \frac{1}{R}\right.$ ) can be ascribed to the higher strain gradients for smaller microwire radii. A size effect due to pile-ups is only observed for microwires with radii smaller than a specific radius for the considered case, this is a radius smaller than $12 \mu \mathrm{m}$. To explain the length of the pile-ups for smaller microwire radii, two different perspectives can be considered: Assuming the same resolution along the radius $r$, the same length of the transition area concerning the drop of the dislocation density (see Fig. 8) takes larger parts of the normalized radius $\frac{r}{R}$ for a smaller micorwire radius $R$. This effect even increases for decreasing microwire sizes and leads to larger pile-ups over the normalized radius. Or, assuming the same resolution along the normalized radius $\frac{r}{R}$, the internal stresses increase compared to the constant external stresses for decreasing microwire radii leading to the larger pile-ups. Since the resulting dislocation microstructure shows a star-like distribution, the plastic slip is influenced by the smoothed pile-up not only inside the microwire at the critical radius $r^{\text {crit }}$ but also near the surface of the microwire.

Comparing the continuum model with DDD results, it is found that the almost linear slope of the dislocation production for a constant torsional rate (see Fig. 12) is consistent to the DDD simulations of $\langle 100\rangle$ oriented single crystalline aluminum microwires applied to a constant torsion rate of $\dot{\varphi}=$ $3 \frac{\circ}{\mu s}$ in [32]. This underlines the qualitative accordance of the amount of dislocation production in the introduced continuum source model for an increasing torsional load.

Regarding experimental analyses of micorwires, most investigations study the misorientation. The qualitative behavior of the misorientation angles and rotation axes shown in Fig. 13 is consistent to the theoretical considerations in Fig. 4. In the cross section of the wire at half of the wire length, where all four preferred slip system $(\mathrm{A} 3, \mathrm{~B} 4, \mathrm{C} 3, \mathrm{D} 4)$ are dominant, the rotation axes point towards the torsion axis. The center point of the wire cross section at half of the wire length keeps nearly its initially orientation. Near the top and the bottom of the wire, the continuum is locally influenced only by specific slip systems since the dislocation density is generated near the surface and move on slip planes, that have a plane normal, which do not align to the torsion axis for a $\langle 100\rangle$ crystal orientation. Thus, each slip system leaves a respective area "untouched" for geometric reasons resulting in an alignment of the rotation axes to the torsion axis. Since the orientation is uniform in the area close to the torsion axis, the continuum elements in the torsion axis show a misorientation compared to its initial configuration near the top and bottom of the wire. Consequently, an hourglass shape can be recognized for the misorientation angle, see Fig. 13. This effect can occur at geometric boundaries of the wire but also at transition regions of high and low dislocation activity. Therefore, it should be noted, that a reduced slip systems consideration using the four slip systems for the resulting plastic distortion in Fig. 3 would not be able to capture this effect.

Comparing the simulations with experimental data, the simulation results showing the misorientation over different cross sections along the torsion axis (Fig. 13) reproduce essential features of the experimental results according to the study presented in [43]. However, it has to be remarked, that the misorientation in the CDD simulation was computed during loading based on the current plastic distortion, whereas the experimental data has been determined ex-situ by measuring the remaining plastic deformation of randomly chosen cross sections. Although this could imply significantly different dislocation microstructures, the presented CDD results and the theoretical considerations suggest that the differences in the misorientation over the cross sections in the experimental results could hint to different regions of slip activity and therefore to a localization of the plastic slip for the microwire shown on the right in Fig. 13. The asymmetric distribution could be traced back to the misalignement in the experimental setup as already discussed in [43]. So for the interpretation of the plastic deformation process, the set of rotation axis, rotation angle and misorientation of the wire center compared to the initial configuration is considered. An interesting aspect is that the interaction of slip systems can lead to a (partly) vanishing misorientation since it only takes the skew symmetric part of the distorsion tensor into account. Accordingly, the equivalent plastic strain is not directly linked to the misorientation in all cases.

Although the introduced CDD formulation shows meaningful results compared to DDD and experimental data, it has to be remarked that the continuum model do not incorporate any discrete nucleation or multiplication events and just account for dislocation reactions in an implicit way by accounting for the resulting strengthening by a dislocation based yield formulation. However, the incorporation of dislocation density based stress interaction terms, including mean-field stress, backstress and yield stress, allows for a physical based representation of the microstructural processes leading to strain hardening in a homogenized way. This is accompanied by the reduction of source activity with increasing dislocation density since the resolved shear stress including the internal stress interaction affects the productivity of the sources. Furthermore, the presented continuum source model neglects the reduced mean free path due to the dislocation pile-up in the calculation of the mean sheared area $A_{s}^{\text {av }}$ (cp. $c^{\text {load }}$ in equation $(19)$ ) and on the the volumetric mean value calculation of the globally expected plastic strain rate $\dot{\varepsilon}^{\text {pl,exp }}$ (cp. c $^{\text {geom }}$ in equation 20 according to Appendix $B$. Consequently, the consideration of the higher increase of the plastic slip for increasing torsional angles in Fig. 
9 could be enhanced by a more precise calculation of $A_{s}^{\text {av }}$ and $\mathrm{c}^{\text {geom }}$. The dislocation density pile-up at the neutral axis as it is represented in the model due to the internal stress interaction leads to a size effect with larger pile-ups for smaller microwire radii. However, an increase of the produced curvature density for smaller micorwires would not affect the different pile-up lengths, but shifts the transition point, defined by the critical radius according to Fig. 8, closer to the surface. Possible model modifications are an adaption of $A_{s}^{\text {av }}$ and c $\mathrm{c}^{\text {geom }}$ or the incorporation of the resulting plastic shear strain into the calculation of the globally expected plastic shear strain per local source activation $\gamma_{s}^{\exp }$ during the running simulation leading to a regulation of the dislocation density production.

\section{Conclusion}

In this paper, a three-dimensional crystal plasticity continuum formulation has been extended by a homogenized, mechanism based dislocation source model. This enabled the physically based analysis of the evolution of the dislocation microstructure for $\langle 100\rangle$ oriented single crystalline microwires under torsion.

The formulation has been tested by the application to simple benchmark systems. According to theoretical considerations and validated by the simulation results, the slip systems for a microwire with $\langle 100\rangle$ crystal orientation can be divided into two groups with respect to the relaxation principle, the amount and alignment of the dislocation density on the slip systems, and the ratio of screw and edge GND densities in the dislocation pile-ups at the neutral axes. It has been found that the contributions of the individual slip systems affect the resulting dislocation microstructure in a characteristic way.

For the wires under torsion, the dislocation density is produced near the surface, driven into the wire according to the stress field and accumulates in the center of the wire forming an inhomogeneous, star-like spatial distribution over the cross section. Consequently, a characteristic dislocation density distribution over the full cross section occurs, which is in accordance to DDD findings. This is a feature that can not be modeled e.g. by the CTT theory or rotational symmetric models since dislocation density is located only in the center of the wire in these formulations. In accordance to the CTT considerations, the results showed a relaxed continuum area in the center of the wire and a nearly constant plastic strain near the surface. Due to the mobility of the dislocation density, the region in the wire center is plastically deformed although the local stress is lower than the critical stress for the source activation. The locality of the transition point is consistent to the CTT for the considered lower loads. However, it slightly differs for higher loads due to the impact of the dislocation pile-up length on the considered source model.

Varying the geometric dimensions of the microwires for a constant torsional load, an increasing dislocation density due to increasing strain gradients is observed for decreasing microwire radii. For microwires with a radius smaller than $12 \mu \mathrm{m}$, a size effect due to the dislocation pile-ups is observed leading to a broader dislocation density distribution. This causes less plastic slip over the normalized radius for smaller microwire radii, whereas microwires with a radius larger than $12 \mu \mathrm{m}$ do not show such a size effect due to the dislocation pile-ups.

For a constant torsional rate, a linear dislocation production rate is found, which is consistent to DDD simulations. The theoretically predicted inhomgeneity of the misorientation along the microwire could be confirmed. Thereby, the relevance of the combination of misorientation angle, rotation axis, and configuration of the reference point for the interpretation of (experimental) results are demonstrated. For the considered loading, the respective rotation axes of the misorientation in the cross section at half of the wire length is pointing towards the center. In contrast, the rotation axes align to the torsion axis at the top and bottom of the wire uniformly over the cross section due to local slip activity of specific slip systems caused by geometric reasons.

\section{Acknowledgement}

We thank Patric Gruber and Michael Ziemann (IAM-WBK, KIT) for the contribution of the experimental results and the interesting discussions. The financial support for this work in the context of the DFG research group FOR1650 'Dislocation based Plasticity' as well as the support by the European Social Fund and the state Baden-Wuerttemberg is gratefully acknowledged. This work was performed on the computational resource ForHLR II funded by the Ministry of Science, Research and the Arts Baden-Württemberg and DFG.

\section{Appendix A. Notation}

Scalars are represented by lowercase letters $\{a, \alpha\}$, vectors and second-order tensors by bold letters $\{\mathbf{a}, \boldsymbol{\alpha}, \mathbf{A}\}$ as well as fourth-order tensors by uppercase blackboard bold letters $\mathbb{A}$. The scalar product is denoted by $\mathbf{a} \cdot \mathbf{b}$, the cross product by $\mathbf{a} \times \mathbf{b}$, the dyadic product by $\mathbf{a} \otimes \mathbf{b}$ and the linear mapping by A $[\mathbf{b}]$. The absolute value is written as $|a|$, the $L^{2}$-Norm as $\|\mathbf{A}\|$, the unit tensor as $\mathbb{1}$, the nabla operator as $\nabla$ and the time derivation as $\dot{a}$. The single slip system are named according to the Schmid-Boas Notation [27] as listed in Tab. A.1.

\begin{tabular}{||c|c|c|c||c|c|c|c||}
\hline \multicolumn{2}{|c|}{ No. } & m & d & \multicolumn{2}{|c|}{ No. } & m & d \\
\hline \hline 1 & B5 & $(111)$ & {$[\overline{1} 10]$} & 7 & D6 & $(\overline{1} 1 \overline{1})$ & {$[110]$} \\
\hline 2 & B4 & $(111)$ & {$[\overline{1} 01]$} & 8 & D1 & $(\overline{1} 1 \overline{1})$ & {$[0 \overline{1} \overline{1}]$} \\
\hline 3 & B2 & $(111)$ & {$[0 \overline{1} 1]$} & 9 & D4 & $(\overline{1} 1 \overline{1})$ & {$[10 \overline{1}]$} \\
\hline \hline 4 & C5 & $(11 \overline{1})$ & {$[\overline{1} 10]$} & 10 & A6 & $(\overline{1} 11)$ & {$[110]$} \\
\hline 5 & C1 & $(11 \overline{1})$ & {$[0 \overline{1} \overline{1}]$} & 11 & A3 & $(\overline{1} 11)$ & {$[101]$} \\
\hline 6 & C3 & $(11 \overline{1})$ & {$[\overline{1} 0 \overline{1}]$} & 12 & A2 & $(\overline{1} 11)$ & {$[0 \overline{1} 1]$} \\
\hline
\end{tabular}

Tab. A.1: Indication of all twelve preferred slip systems for $f c c$ metals using the Schmid-Boas notation. 


\section{Appendix B. Geometric correction factor}

The arithmetical average of a linear distribution over a line is equal to half of the boundary value, whereas the arithmetical average of a circle with a linear distribution along the radius $r$ is equal to two third of the boundary value:

Average along a line: $\varepsilon_{\mathrm{gl}}=\frac{\frac{1}{2} * R * \varepsilon_{r=R}}{R}=\left.\frac{1}{2} \varepsilon\right|_{r=R}$.

Average of a circle: $\varepsilon_{\mathrm{gl}}=\frac{\left.\pi * R^{2} * \varepsilon\right|_{r=R}-\left.\frac{1}{3} * \pi * R^{2} * \varepsilon\right|_{r=R}}{\pi * R^{2}}=\left.\frac{2}{3} \varepsilon\right|_{r=R}$.

In the present considerations, the mean value calculation using the distribution over the cross section is mapped to the mean value calculation referring to the distribution over the radius. This motivates a geometric factor of $c^{\text {geom } \frac{2}{3}}=\frac{1}{2} \Leftrightarrow c^{\text {geom }}=\frac{3}{4}$ for the global strain calculation by the mean value of the local strains if the torsion of a circular cross-section is considered. In contrast to the local strain rate $\dot{\varepsilon}$, the globally expected plastic strain rate due to the local dislocation production $\dot{\varepsilon}^{\text {pl,exp }}$ affects the entire system on the slip plane in radial direction due to the dislocation motion. Therefore, no geometric factor is needed for the mean value calculation of the globally expected plastic strain rate.

\section{Appendix C. Calculated Quantities}

The equivalent strain in calculated analogously to the von Mises stress as $\varepsilon_{e q}=\sqrt{\frac{2}{3}}\left\|\varepsilon^{\prime}\right\|=\sqrt{\frac{2}{3}}\left\|\boldsymbol{\varepsilon}-\frac{1}{3} \operatorname{trace}(\boldsymbol{\varepsilon}) \mathbb{1}\right\|$.

Taking the aspect ratio of the microwire $\frac{R}{L}$ with a wire length $\mathrm{L}$ into account, the relation between the surface shear $\gamma_{R}$ and the torsion angle $\varphi$ reads for small torsion angles: $\gamma_{R}=\frac{\varphi}{L} R$.

To show the material response independent of the geometric dimensions, the torque is normalized by the third power of the wire radius:

$M_{T}^{\text {norm }}=\frac{M_{T}}{R^{3}}$ with $M_{T}=\int \tau_{x y} *\left(z-c_{z}\right)-\tau_{x z} *\left(x-c_{x}\right) d A_{y}$. Here describes $\left\{c_{x}, c_{z}\right\}$ the position vector of the torsion axis in the cross section of the wire.

\section{Appendix D. Misorientation from distorsion fields}

The infinitesimal rotation tensor $\mathbf{W}$ represents the skew symmetric part of the distorsion tensor $\boldsymbol{\beta}: \mathbf{W}=\operatorname{skw}(\boldsymbol{\beta})=\frac{1}{2}\left(\boldsymbol{\beta}-\boldsymbol{\beta}^{T}\right)$. Since it consist of three independent components, it can be interpreted as an rotation of the angle $\alpha$ around the normalized rotation axis $\mathbf{n}^{\text {rot }}=\left(n_{x}^{\text {rot }} n_{y}^{\text {rot }} n_{z}^{\text {rot }}\right)^{T}$ describing the rotation from the initial configuration into the actual one:

$$
\mathbf{W}=\alpha\left(\begin{array}{ccc}
0 & n_{z}^{\text {rot }} & -n_{y}^{\text {rot }} \\
-n_{z}^{\text {rot }} & 0 & n_{x}^{\text {rot }} \\
n_{y}^{\text {rot }} & -n_{x}^{\text {rot }} & 0
\end{array}\right) \quad, \quad \alpha=\left\|\alpha \mathbf{n}^{\text {rot }}\right\| .
$$

The resulting rotation matrix reads

$$
\mathbf{R}=\cos (\alpha) \mathbb{1}+(1-\cos (\alpha))\left(\mathbf{n}^{\mathrm{rot}} \otimes \mathbf{n}^{\mathrm{rot}}\right)+\sin (\alpha) \frac{1}{\alpha} \mathbf{W}^{T} .
$$

The misorientation describes the misalignment compared to a reference configuration, like the initial configuration or the center of the wire. Therefore, both the rotation matrix $\mathbf{R}$ at each site and the rotation matrix $\mathbf{R}^{\text {ref }}$ of the reference continuum are necessary to calculate the misorientation angle $\theta$ between the considered continuum and the reference continuum:

$$
\begin{gathered}
\theta=\arccos \left(\frac{\operatorname{trace}\left(\mathbf{R}^{\mathrm{mis}}\right)-1}{2}\right) \quad, \quad \mathbf{R}^{\mathrm{mis}}=\mathbf{R}^{\mathrm{ref}} \mathbf{R}^{-1} \\
\mathbf{n}^{\mathrm{mis}}=\frac{1}{2 \sin (\theta)}\left(\begin{array}{c}
R_{y z}^{\mathrm{mis}}-R_{z y}^{\mathrm{mis}} \\
R_{z x}^{\mathrm{mis}}-R_{x z}^{\mathrm{mis}} \\
R_{x y}^{\mathrm{mis}}-R_{y x}^{\mathrm{mis}}
\end{array}\right) .
\end{gathered}
$$

If the reference configuration is identical to the initial configuration, the following relations are valid: $\mathbf{R}^{\text {ref }}=\mathbb{1}, \theta=\alpha$ and $\mathbf{n}^{\text {mis }}=\mathbf{n}^{\text {rot }}$.

\section{Appendix E. Rotated crystal orientation}

The resulting dislocation microstructure for a single crystalline fcc microwire described in Fig. 1 1 under constant torsion angle of $\varphi=1^{\circ}$ is shown in Fig. E.14. Here, the $\langle 100\rangle$ crystal orientation described in Tab. A.1 is rotated by $22.5^{\circ}$ around the torsion axis.
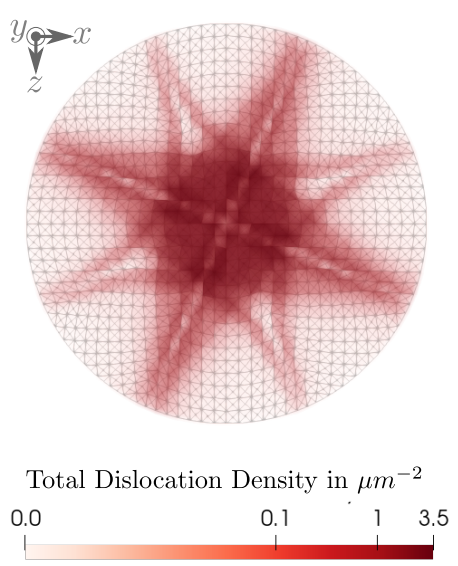

Fig. E.14: Total dislocation density close to the end of the relaxation step. The dislocation density has not reached its equilibrium state yet, but a star-like dislocation density structure is already observe-able.

\section{Appendix F. Reference value for the source length}

The resulting stress-strain curves for various sample sizes of single crystalline fcc microwires according to the system shown in Fig 1 under constant torsional rate $\left(+0.5 \frac{\circ}{\mu s}\right)$ are shown in Fig F.15. The reference value for the source length (i.e. $l_{s}^{\text {ref }}$ in equation (10) is an input parameter of the continuum simulation, which has to be chosen e.g. according to experimental results, and directly impacts the initiation of yielding in the numerical formulation. Here, for the micorwires with a radius of $R=\{12,6,3\} \mu \mathrm{m}$, the reference value for the source length is chosen to $l_{s}^{\text {ref }}=\{0.32,0.22,0.15\} \mu m$ referring to a critical source stress of $\tau_{s}^{\text {crit }}=\{24,35,49\} M P a$ (according to experimental results presented in [39]). 


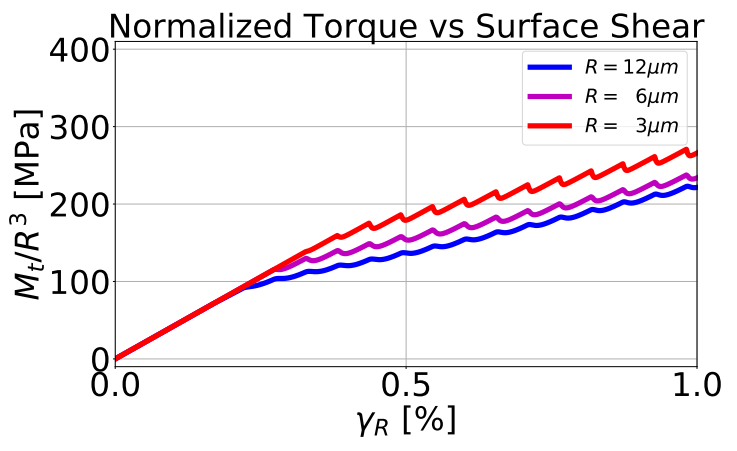

Fig. F.15: Normalized torsion moments are plotted versus the surface shear for a varying reference value for the source length depending on the microwire radius.

\section{Appendix G. Comparison to DDD results}

Discrete dislocation dynamics simulation of $1 \mu \mathrm{m}$ thick copper micorwires under torsion for a $\langle 100\rangle$ crystal orientation showing a resulting star-like distribution of the dislocations.

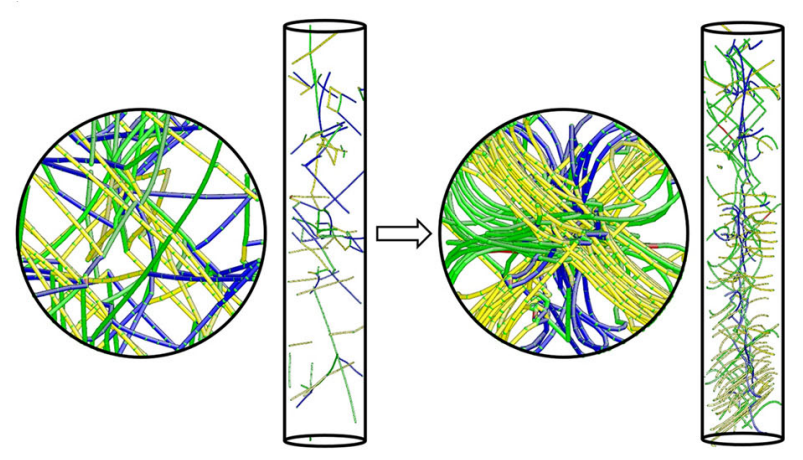

Fig. G.16: Dislocation microstructure evolution of a $\langle 100\rangle$ oriented $1 \mu \mathrm{m}$ pillar extracted from [26]

\section{References}

\section{References}

[1] Arsenlis, A., Parks, D., 1999. Crystallographic aspects of geometricallynecessary and statistically-stored dislocation density. Acta materialia 47, 1597-1611. URL:https://doi.org/10.1016/S1359-6454(99) 00020-8

[2] Bardella, L., Panteghini, A., 2015. Modelling the torsion of thin metal wires by distortion gradient plasticity. Journal of the Mechanics and Physics of Solids 78, 467-492. URL: https://doi.org/10.1016/ j.jmps.2015.03.003

[3] Bayerschen, E., Prahs, A., Wulfinghoff, S., Ziemann, M., Gruber, P., Walter, M., Böhlke, T., 2016. Modeling contrary size effects of tensileand torsion-loaded oligocrystalline gold microwires. Journal of materials science 51, 7451-7470. URL: https://doi.org/10.1007/ s10853-016-0020-7

[4] Dunstan, D., 2012. Critical thickness theory applied to micromechanical testing. Advanced Engineering Materials 14, 942-947. URL: https : //doi.org/10.1002/adem. 201200017

[5] Dunstan, D.J., Bushby, A., 2004. Theory of deformation in small volumes of material. Proceedings of the Royal Society of London. Series A: Mathematical, Physical and Engineering Sciences 460, 2781-2796. URL: https://doi.org/10.1098/rspa.2004.1306
[6] El-Awady, J.A., Wen, M., Ghoniem, N.M., 2009. The role of the weakestlink mechanism in controlling the plasticity of micropillars. Journal of the Mechanics and Physics of Solids 57, 32-50. URL: https://doi.org/ 10.1016/j.jmps.2008.10.004

[7] Fleck, N., Muller, G., Ashby, M., Hutchinson, J., 1994. Strain gradient plasticity: theory and experiment. Acta Metallurgica et materialia 42, 475-487. URL: https://doi.org/10.1016/0956-7151(94) 90502-9

[8] Greer, J.R., Oliver, W.C., Nix, W.D., 2005. Size dependence of mechanical properties of gold at the micron scale in the absence of strain gradients. Acta Materialia 53, 1821-1830. URL: https ://doi.org/10.1016/j . actamat.2004.12.031

[9] Groma, I., Csikor, F., Zaiser, M., 2003. Spatial correlations and higherorder gradient terms in a continuum description of dislocation dynamics. Acta Materialia 51, 1271-1281. URL: https://doi.org/10.1016/ S1359-6454(02) 00517-7

[10] Gudmundson, P., 2004. A unified treatment of strain gradient plasticity. Journal of the Mechanics and Physics of Solids 52, 1379-1406. URL: https://doi.org/10.1016/j.jmps.2003.11.002

[11] Hirth, J.P., Lothe, J., 1982. Theory of dislocations. A Wiley-Interscience Publication. 2. ed. ed., Wiley, New York, NY [u.a.].

[12] Hochrainer, T., Sandfeld, S., Zaiser, M., Gumbsch, P., 2014. Continuum dislocation dynamics: towards a physical theory of crystal plasticity. Journal of the Mechanics and Physics of Solids 63, 167-178. URL: https://doi.org/10.1016/j.jmps.2013.09.012

[13] Kaluza, M., Le, K., 2011. On torsion of a single crystal rod. International Journal of Plasticity 27, 460-469. URL: https ://doi.org/10.1016/ j.ijplas.2010.07.003

[14] Le, K.C., Piao, Y., 2016. Distribution of dislocations in twisted bars. International Journal of Plasticity 83, 110-125. URL: https://doi. org/10.1016/j.ijplas.2016.04.006

[15] Le, K.C., Piao, Y., 2019. Thermodynamic dislocation theory: Size effect in torsion. International Journal of Plasticity 115, 56-70. URL: https : //doi.org/10.1016/j.ijplas.2018.11.009

[16] Li, D., Zbib, H., Sun, X., Khaleel, M., 2014. Predicting plastic flow and irradiation hardening of iron single crystal with mechanism-based continuum dislocation dynamics. International Journal of Plasticity 52, 3-17. URL:https://doi.org/10.1016/j.ijplas.2013.01.015

[17] Liu, D., He, Y., Dunstan, D.J., Zhang, B., Gan, Z., Hu, P., Ding, H., 2013. Toward a further understanding of size effects in the torsion of thin metal wires: an experimental and theoretical assessment. International Journal of Plasticity 41, 30-52. URL: https : //doi .org/10.1016/j . ijplas.2012.08.007

[18] Liu, D., Zhang, X., Li, Y., Dunstan, D., 2018. Critical thickness phenomenon in single-crystalline wires under torsion. Acta Materialia 150, 213-223. URL: https://doi.org/10.1016/j.actamat.2018.03. 022

[19] Madec, R., Devincre, B., Kubin, L., 2002. From dislocation junctions to forest hardening. Physical review letters 89, 255508. URL: https : //doi.org/10.1103/PhysRevLett.89.255508

[20] Mason, W.P., Rosenberg, A., 1966. Phonon and electron drag coefficients in single-crystal aluminum. Physical Review 151, 434. URL: https: //doi.org/10.1103/PhysRev.151.434

[21] Monavari, M., Zaiser, M., 2018. Annihilation and sources in continuum dislocation dynamics. Materials Theory 2, 3. URL: https://doi.org/ 10.1186/s41313-018-0010-z

[22] Oh, S.H., Legros, M., Kiener, D., Dehm, G., 2009. In situ observation of dislocation nucleation and escape in a submicrometre aluminium single crystal. Nature materials 8, 95-100. URL: https://doi.org/10. 1038/nmat2370

[23] Orowan, E., 1934. Zur kristallplastizität. Zeitschrift für Physik 89, 605659. URL: https://doi.org/10.1007/BF01341479

[24] Parthasarathy, T.A., Rao, S.I., Dimiduk, D.M., Uchic, M.D., Trinkle, D.R., 2007. Contribution to size effect of yield strength from the stochastics of dislocation source lengths in finite samples. Scripta Materialia 56, 313-316. URL: https://doi.org/10.1016/j.scriptamat.2006. 09.016

[25] Roters, F., 2003. A new concept for the calculation of the mobile dislocation density in constitutive models of strain hardening. physica status solidi (b) 240, 68-74. URL: https://doi.org/10.1002/pssb. 200301873 
[26] Ryu, I., Cai, W., Nix, W.D., Gao, H., 2016. Anisotropic size-dependent plasticity in face-centered cubic micropillars under torsion. JOM 68, 253 260. URL: https://doi.org/10.1007/s11837-015-1692-1

[27] Schmid, E., Boas, W., 1935. Kristallplastizität : mit besonderer Berücksichtigung der Metalle. Struktur und Eigenschaften der Materie in Einzeldarstellungen ; 17, Springer, Berlin. URL: https://link. springer.com/book/10.1007/978-3-662-34532-0

[28] Schmitt, S., Gumbsch, P., Schulz, K., 2015. Internal stresses in a homogenized representation of dislocation microstructures. Journal of the Mechanics and Physics of Solids 84, 528-544. URL: https://doi. org/10.1016/j.jmps.2015.08.012

[29] Schmitt, S., Stricker, M., Gumbsch, P., Schulz, K., 2019. A mechanismbased homogenization of a dislocation source model for bending. Acta Materialia 164, 663-672. URL: https://doi.org/10.1016/j . actamat.2018.11.013

[30] Schulz, K., Dickel, D., Schmitt, S., Sandfeld, S., Weygand, D., Gumbsch, P., 2014. Analysis of dislocation pile-ups using a dislocation-based continuum theory. Modelling and Simulation in Materials Science and Engineering 22,025008. URL: https://doi.org/10.1088/0965-0393/ 22/2/025008

[31] Schulz, K., Wagner, L., Wieners, C., 2019. A mesoscale continuum approach of dislocation dynamics and the approximation by a runge-kutta discontinuous galerkin method. International Journal of Plasticity 120 , 248-261. URL: https://doi.org/10.1016/j.ijplas.2019.05. 003

[32] Senger, J., Weygand, D., Kraft, O., Gumbsch, P., 2011. Dislocation microstructure evolution in cyclically twisted microsamples: a discrete dislocation dynamics simulation. Modelling and Simulation in Materials Science and Engineering 19, 074004. URL: https://doi .org/10. 1088/0965-0393/19/7/074004

[33] Sudmanns, M., Gumbsch, P., Schulz, K., 2018. Plastic flow and dislocation strengthening in a dislocation density based formulation of plasticity. Computational Materials Science 151, 317-327. URL: https : //doi.org/10.1016/j.commatsci.2018.04.065

[34] Sudmanns, M., Stricker, M., Weygand, D., Hochrainer, T., Schulz, K., 2019. Dislocation multiplication by cross-slip and glissile reaction in a dislocation based continuum formulation of crystal plasticity. Journal of the Mechanics and Physics of Solids 132, 103695. URL: https://doi . org/10.5445/IR/1000098013

[35] Tsagrakis, I., Konstantinidis, A., Aifantis, E., 2003. Strain gradient and wavelet interpretation of size effects in yield and strength. Mechanics of materials 35, 733-745. URL: https://doi.org/10.1016/ S0167-6636(02)00205-3

[36] Weertman, J., 2002. Anomalous work hardening, non-redundant screw dislocations in a circular bar deformed in torsion, and non-redundant edge dislocations in a bent foil. Acta Materialia 50, 673-689. URL: https : //doi.org/10.1016/S1359-6454(01)00405-0

[37] Weinberger, C.R., Cai, W., 2009. Orientation-dependent plasticity in metal nanowires under torsion: Twist boundary formation and eshelby twist. Nano letters 10,139-142. URL: https://doi.org/10.1021/ nl903041m

[38] Weinberger, C.R., Cai, W., 2010. Plasticity of metal wires in torsion: Molecular dynamics and dislocation dynamics simulations. Journal of the Mechanics and Physics of Solids 58, 1011-1025. URL: https:// doi.org/10.1016/j.jmps.2010.04.010

[39] Wu, J., Tsai, W., Huang, J., Hsieh, C., Huang, G.R., 2016. Sample size and orientation effects of single crystal aluminum. Materials Science and Engineering: A 662, 296-302. URL: https://doi.org/10.1016/j . msea.2016.03.076

40] Zhang, H., Dong, S.x., Zhang, S.y., Wang, T.h., Zhang, Z.n., Fan, L., 2006. Ultrasonic micro-motor using miniature piezoelectric tube with diameter of $1.0 \mathrm{~mm}$. Ultrasonics 44, e603-e606. URL: https://doi. org/10.1016/j.ultras.2006.05.064

[41] Zhu, T., Bushby, A., Dunstan, D., 2008. Materials mechanical size effects: a review. Materials Technology 23, 193-209. URL: https://doi.org/ 10.1179/175355508X376843

[42] Zhu, Y., Wang, H., Zhu, X., Xiang, Y., 2014. A continuum model for dislocation dynamics incorporating frank-read sources and hall-petch relation in two dimensions. International Journal of Plasticity 60, 19-39. URL: https://doi.org/10.1016/j.ijplas.2014.04.013

[43] Ziemann, M., Chen, Y., Kraft, O., Bayerschen, E., Wulfinghoff, S., Kirch- lechner, C., Tamura, N., Böhlke, T., Walter, M., Gruber, P., 2015. Deformation patterns in cross-sections of twisted bamboo-structured au microwires. Acta Materialia 97, 216-222. URL: https://doi.org/10. 1016/j.actamat.2015.06.012 